\title{
1 A survey of optimal strategy for signature-based drug 2 repositioning and an application to liver cancer
}

3 Chen Yang $^{1 \#}$, Mengnuo Chen ${ }^{1 \#}$, Siying Wang ${ }^{1 \#}$, Ruolan Qian ${ }^{1}$, Xiaowen Huang ${ }^{2}$, Jun

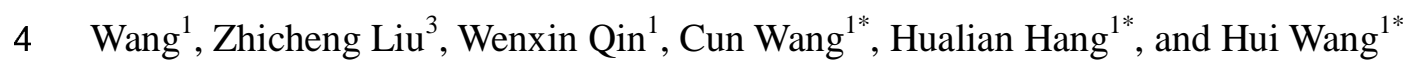

5 *Corresponding author. Email: hwang@shsci.org (H.W.), hanghualian@shsmu.edu.cn

6 (H.H.), and cwang@ shsci.org (C.W.)

$7 \quad{ }^{\#}$ These authors contributed equally to this work.

$8 \quad{ }^{1}$ State Key Laboratory of Oncogenes and Related Genes, Shanghai Cancer Institute \& 9 Department of Liver Surgery, Renji Hospital, Shanghai Jiao Tong University School 10 of Medicine, Shanghai, China.

$11{ }^{2}$ Key Laboratory of Gastroenterology and Hepatology, Division of Gastroenterology 12 and Hepatology, Renji Hospital, Shanghai Jiao Tong University School of Medicine, 13 Shanghai, China.

$14{ }^{3}$ Hepatic Surgery Center, Tongji Hospital, Tongji Medical College, Huazhong 15 University of Science and Technology, Wuhan, China.

\section{Keywords}

18 Drug prediction, Connectivity map, LINCS, Liver cancer, Homoharringtonine 


\section{Abstract}

21 Pharmacologic perturbation projects, such as Connectivity Map (CMap) and Library 22 of Integrated Network-based Cellular Signatures (LINCS), have produced many 23 perturbed expression data, providing enormous opportunities for computational 24 therapeutic discovery. However, currently there is no consensus on which 25 methodologies and parameters are the most optimal to conduct such analysis. Aiming 26 to fill this gap, we developed new benchmarking standards for quantitatively 27 estimating drug retrieval performance. Investigations of potential factors influencing 28 drug retrieval were conducted based on these standards. As a result, we determined an 29 optimal strategy for LINCS data-based therapeutic discovery. With this approach, we 30 further identified new therapeutics for liver cancer of which the current treatment 31 modalities remain imperfect. Both computational and experimental results 32 demonstrated homoharringtonine (HHT) could be a promising anti-liver cancer agent. 33 In summary, our findings will not only impact the future applications of LINCS data 34 but also offer new opportunities for therapeutic intervention for liver cancer. 


\section{Introduction}

Despite the major advances in drug research and development (R\&D), the cost for $d e$ novo drug development remains high, ranging from $\$ 3$ billion to more than $\$ 30$ billion. Moreover, it usually takes over 10 years to bring a new drug from bench to bedside, reflecting the complex challenges in this area [1]. Within this context, exploring new indications for existing drugs (drug-centric) or identifying effective drugs for certain disease (disease-centric) represent an appealing concept, namely 'drug repositioning' (or 'drug repurposing'), that can greatly shorten the gap between preclinical drug research and clinical applications [2]. Leveraging big data-driven approaches, drug repositioning can be performed computationally, which has the potential to complement traditional therapeutic discovery means and further improve the cost-effectiveness of drug development [3]. The most notably data resources supporting the in silico-based therapeutic discovery campaigns would be the Connectivity Map (CMap) [4] and its recent extension called Library of Integrated Network-Based Cellular Signatures (LINCS) [5]. These two projects have generated large-scale drug-induced gene expression profiles on multiple cancer cell lines under different treatment conditions (CMap Build 2: 3 cell lines, 1,309 compounds; LINCS: 77 cell lines, 19,811 compounds), representing a treasure trove for in silico therapeutic exploration [6]. As a 1,000-fold scale-up of the original CMap, LINCS contained dramatic increases in both cell line types and perturbations, making it the focus of the present investigation.

The computational drug discovery approach using LINCS (also CMap) data is based upon the basic concept called 'signature reversion' [3]. Specifically, to identify candidate agents for treating a certain disease, molecular signature characterizing the states of this disease needs to be generated first. Then, through comparing compound signatures with disease signatures, inverse compound-disease relationships can be identified. Compounds with the ability to reverse disease-specific gene expression pattern are considered as therapeutic candidates (Fig. 1). Several successful applications of this approach include the discovery of rapamycin as combination 
partner of glucocorticoids in treating lymphoid malignancies [7]; topiramate as a potential agent against inflammatory bowel disease [8]; citalopram as a therapeutic option for patients with metastatic colorectal cancer [9]; niclosamide and pyrvinium pamoate as potential therapeutics for liver cancer [10]. Despite its success, there are still many unsolved problems with this approach. Currently, due to the lack of appropriate benchmarking standards, limited studies have focused on investigating the potential factors that influence this computational strategy, let alone quantitatively evaluating whether these factors affect the accuracy of drug prediction. Therefore, across current signature reversion studies, no consensus regarding the implementation details has been reached. Constructing the rational benchmarking standards and developing the best practice approach can facilitate the development of computational pharmacology area and help to identify more effective therapeutic strategies for refractory diseases.

Herein we mainly focused on the disease of liver cancer. As one of the most lethal malignancies worldwide, liver cancer directly accounts for nearly one million deaths each year (8.2\% of new cases of cancer-related death) [11]. Hepatocellular carcinoma (HCC) is the major type of liver cancer, representing approximately $90 \%$ of all liver cancer cases [12]. Although many standard of care therapies, including Lenvatinib [13], regorafenib [14], cabozantinib [15], ramucirumab [16], pembrolizumab [17], nivolumab [18] and atezolizumab-bevacizumab [19], have been approved for treating HCC in recent years, most of them can yield only marginal survival benefit. Thus, more effective therapeutics treatments for HCC are highly desired. The objectives of the present study were threefold. The first objective was to develop novel benchmarking standards for evaluating drug retrieval performance. The second one was to determine the best practice approach for LINCS data-based computational drug repositioning. For the last objective, through exploiting the findings from the second objective, we aimed to identify novel drug candidates against liver cancer. 
94

\section{Results}

\subsection{Summary of influencing factors and compound experiments in LINCS}

Many factors may affect the accuracy of signature-based drug retrieval. We have categorized these factors into three main aspects: acquisition of compound signature (reference signature), generation of disease signature (query signature), and selection of disease-compound matching methods (Additional file 2: Figure S1). Although all factors were mentioned and discussed, not all of them were included into the present analyses, considering that some factors have been covered elsewhere and some were challenging to explore due to data and method restrictions. In this study, systematic analyses were carried out to assess the influences of four major factors on signature matching-based drug discovery, including source of cell line, clinical phenotype of query signature, query signature size, and signature matching method. Detailed descriptions and discussions about all included factors were stated in Additional file 1: Supplementary Discussion.

Since only compound-induced expression data was the focus of this study, we first excluded experiments of other perturbagens, including gene knockdown (or knockout) and gene overexpression manipulations. Subsequently, the distribution of compound profiles was visualized based on their perturbation time, perturbation dose, and cell line used. Most of the measurements were made in the treatment durations of 6h $(43 \%)$ or $24 \mathrm{~h}(56.6 \%)$, and under the concentrations of $5 \mu \mathrm{M}(21 \%)$ or $10 \mu \mathrm{M}(63 \%)$ (Additional file 2: Figure S2a). The count distribution of all cell lines in LINCS was also presented in Additional file 2: Figure S2b. Although 71 cell lines were included in LINCS project in total, not all of them were extensively profiled, and only nine cell lines contained more than 5,000 profiles, which, however, account for $77.8 \%$ of all compound profiles. There were 2,912 compounds shared by these nine cell lines. We further integrating annotation of the most profiled cell lines with treatment duration and concentration information and illustrated the specific profile numbers of each cell line under the conditions of certain time and dose (Additional file 2: Figure S2c). Notably, all the following analyses were performed using LINCS data based on a 
123 fixed perturbation condition of $10 \mu \mathrm{M}$ for 6 hours. Besides, compound profiles of all

124 top nine cell lines were only utilized when investigating the factor 'Source of cell

125 line'. In other cases, we focused exclusively on the cell line of HepG2, as in this study,

126 our main point was to uncover novel therapeutics for liver cancer. A systematic

127 summary of included datasets for analyses was presented in Additional file 3: Table

$128 \mathrm{~S} 1$ and $\mathrm{S} 2$.

\subsection{Cell-type specific gene expression changes induced by compound treatments}

131 Some previous studies utilized compound profiles from cell lines irrelevant to the 132 disease their studied for performing signature-based drug repositioning. To investigate 133 whether this was a reasonable practice, we conducted following analyses based on the 134 LINCS data of the nine most profiled cell lines. First, we visualized the compound 135 profiles in a cosine distance-based two-dimensional t-distributed stochastic neighbor embedding (t-SNE) plot that represented the overall compound perturbation space wherein each dot was equivalent to a unique perturbation and each cell line was color-coded (Fig. 2a). As shown in the figure, most dots with the same color clustered together, indicating that most of compound-induced gene expression changes tended to be cell-type specific. Intriguingly, dots with different colors in the white region seemed to mix together, suggesting that some certain compounds might induce similar gene expression changes across cell lines. To figure out which compounds were likely to cause cell-type specific gene expression changes, and which tended to induce universal changes independent of cell lines, we calculated the pairwise cosine similarities (L1) among the profiles from the same compounds measured in different cell lines (Fig. 2b). The cosine similarity measures range from -1 to 1 , where higher values indicate increased similarity. The similarity scores (compound-level, L2) of the 2,912 unique compounds were determined by calculating the median pairwise cosine similarity values (L1) across the most profiled nine cell lines mentioned before

150 (Additional file 3: Table S3). As a result, a high degree of cell-specificity was 
152 Furthermore, we retrieved the mechanism of action (MOA) information and mapped

153 them to the compounds to determine the MOA-level similarity scores (L3). L3

154 similarity scores were calculated based on the median values of L2 similarity scores

155 of compounds within the same MOA. Results suggested that inhibitors targeting core

156 cellular processes (e.g., cell cycle, RNA transcription, protein synthesis) tended to

157 induce similar changes across all cell lines, generally in agreement with previous

158 findings (Fig. 2d and Additional file 3: Table S3) [5, 20]. We then marked the dots

159 representing the compounds of top five MOAs in the t-SNE plot. As might be

160 expected, most of marked dots fell in clusters within the nonspecific region (Fig. 2e).

161 Apart from investigating similarity of perturbed expression profiles at compound

162 level, we sought to further investigate the cell line pair/cell line-level similarity. Nine

163 cell lines contributed a total of 36 unique cell line pairs. The cell line pair-level

164 perturbed expression similarities (L4) were determined through calculating the

165 median value of similarity scores of all compound pairs between two cell lines, and

166 the corresponding basal expression similarities were computed using Spearman

167 ranked correlation on expression data from CCLE project (Fig. 2f). Based on these 36

168 cell line pairs, we found that there was a significant, albeit not very remarkable,

169 association between the perturbed expression similarities (cell line pair-level, L4) and

170 basal expression similarities (rho $=0.344 ; P=0.040$ ), suggesting that cell lines with

171 similar molecular features were more likely to have consistent gene expression

172 changes upon perturbation (Fig. 2g). Similarities within the nine cell lines were also

173 explored (cell line-level, L5). Among the nine cell lines we tested, PC3 cell line

174 showed the highest L5 similarity score (median value $=0.122)($ Fig. $2 \mathrm{~h})$. Notably, the

175 cosine similarity of 0.122 still denoted a weak relationship, which further supported

176 the conclusion that compound-induced gene expression changes were highly cell line 177 specific.

178 Among the nine most profiled cell lines, HepG2 was the only one derived from 179 liver. To investigate whether HepG2 was an appropriate cell line model for 180 computational therapeutics discovery for liver cancer or other liver-associated 
diseases, we calculated the expression correlation between HepG2 and other cell lines (921 CCLE cell lines) or tissues (17,382 normal tissues from GTEx and 9,701 tumor tissues from TCGA PanCancer). Compared to other tissue-derived cancer cell lines or normal/tumor tissues, HepG2 exhibited a significantly higher expression correlation with liver cancer cell lines (median correlation coefficient $=0.729$ ), normal liver tissues (median correlation coefficient $=0.616$ ), and liver cancer tissues (median correlation coefficient $=0.631)($ Additional file 2: Figure S3). Coupled with above finding of the significant relationship between perturbed expression similarities and basal expression similarities, we supposed that the use of LINCS-derived HepG2 data was preferable to be limited within liver diseases.

\subsection{Developing new benchmarking standards}

Owing to the lack of benchmarking standards, accurate assessment of retrieval performance of signature matching methods remains challenging. Inspired by previous findings $[10,21]$, we proposed two novel benchmarking standards, namely area under curve (AUC)-based standard and Kolmogorov-Smirnov (KS) statistic-based standard. They were built upon different notions and thus independent of each other, which helped to avoid potential bias introduced by single standard. The corresponding benchmarking datasets were developed mainly based on preclinical/clinical data of liver cancer. Detailed processes regarding data collection and metrics calculation have been described in Methods and visualized in Additional file 2: Figure S4a.

The development of AUC-based standard was based on the finding that there existed correlation between the reversal potency and treatment efficacy, which has been reported by several previous studies [10b, 21a]. In order to further validate whether this correlation remained significant in other conditions, we retrieved drug response data from CTRP dataset in which area under the dose-response curve (AUDRC) values were used as measurements of drug sensitivity, and utilized two different HCC signatures as query signatures to obtain KS-based similarity scores [10]. 
210 A total of 109 compounds shared by two datasets were selected to conduct correlation

211 analyses. As a result, statistically significant correlation could still be observed

212 between similarity scores and AUDRC values in these scenarios, further proving the

213 reliability of this standard (Additional file 2: Figure S4b). A benchmark dataset was

214 then generated, composing of 117 unique compounds with both LINCS and drug

215 efficacy $\left(\mathrm{IC}_{50}\right)$ data available, which was taken as a basis for the application of

216 AUC-based standard (Additional file 2: Table S4). The resultant AUC from this

217 standard were termed as drug retrieval-associated AUC (DR-AUC). Higher DR-AUC

218 value indicated better performance.

219 As for KS statistic-based standard, we assumed that agents under evaluation in 220 clinical trial for HCC treatment, namely HCC agents, might possess an increased 221 reversal capacity [10a]. In other words, HCC agents were more likely to cause 222 negative enrichment in KS test. To verify this hypothesis, we compiled a set of 27 223 potential HCC agents which were both included in LINCS and under clinical trials for 224 liver cancer treatment. Besides, similarity scores of all compounds tested in HepG2 225 were also calculated, which were then used as ranked list for KS test. The results of 226 KS test demonstrated the above assumption that the HCC agent set was negatively 227 enriched significantly (Additional file 2: Figure S4c). Accordingly, KS statistic-based 228 standard could be implemented based on this 27-agent set and the resultant 229 enrichment scores (ES) here were termed as drug retrieval-associated ES (DR-ES) 230 (Additional file 3: Table S5). Of note, in contrast to DR-AUC, lower DR-ES values 231 denoted better performance.

232

\section{2.4.Benchmarking signature matching methods for drug retrieval}

234 These two independent benchmarking standards enabled us to quantitatively assess 235 the retrieval performance of different signatures matching methods and thus aided in 236 the identification of optimal methodology for computational drug repositioning. In 237 total, six available methods including eXtreme Sum (XSum) [21b], eXtreme Cosine 238 (XCos) [21b, 22], eXtreme Pearson (XCor) [23], eXtreme Spearman (XSpe) [23], 

[10b] were included for performance comparison. To minimize technical bias introduced by different query signatures, four HCC signatures with different sizes generated from distinct datasets were utilized for benchmarking (Additional file 3: Table S6). Of these, $\mathrm{Sig}_{\text {gastro }}[10 \mathrm{a}]$ and $\mathrm{Sig}_{\mathrm{NC}}[10 \mathrm{~b}]$ were directly obtained from previously publications, while $\mathrm{Sig}_{\mathrm{LIRI}}$ and $\mathrm{Sig}_{\mathrm{GSE} 54236}$ were generated using RNAseq data from LIRI cohort and microarray data from GSE54236, respectively. A brief summary of above essential components involved in the evaluation process was illustrated in Fig. 3a.

Considering that the performance of eXtreme methods (including XSum, XCos, XCor, and XSpe) may be affected by the number of top genes (topN), we thus calculated the DR-AUC or DR-ES values of each eXtreme methods iteratively, using topN ranging from 50 to 489 . In the condition of using $\operatorname{Sig}_{\text {LIRI }}$ as query signature, both benchmarking standards demonstrated that XSum outperformed other five methods across almost all candidate topNs (Fig. 3b). Concordantly, when using other three query signatures, XSum also achieved better performance compared with other methods, except in the case of using $\operatorname{Sig}_{\text {Gastro }}$ as query signature and AUC-based standard for benchmarking, where RGES showed a similar performance with XSum (Additional file 2: Figure S5a-c). Generally, XSum exhibited a consistently satisfactory performance, independently of the query signature and benchmarking standard, and thus could be used as the optimal methodology for drug retrieval (Additional file 3: Table S7 and S8). In addition, our analyses also demonstrated that the recently developed RGES (a modification of the KS method) appeared superior to the KS method in most cases and might serve as an alternative approach for KS-based connectivity mapping [10b].

Since we have proved that XSum could be used as the optimal matching method, we next sought to find the most appropriate topN value for applying XSum method to achieve the best retrieval performance. Directly selecting the exact topN value where 
268

269

270

271

272

273

274

275

276

277

278

279

280

281

282

283

284

285

286

287

288

289

290

291

292

293

294

295

296

and could be prone to over fitting. Given the continuous trait of candidate topNs, we chose to divide them into several smaller groups, typically 50 topN values in each group. The DR-AUC/DR-ES of a certain group was defined as the mean DR-AUC/DR-ES values within this group, which could decrease potential influences brought about by the outliers. With this normalization approach, relatively consistent results across all conditions could be obtained. The optimal topN group which yielded best performance were either 'top150-200' or 'top200-250' groups (Additional file 3: Table S9). Besides, we also observed a biphasic pattern of fitting curves, with the inflection points appearing where topNs were around 200 (Fig. 3c and Additional file 2: Figure S5d-f). Based on these results, we supposed that topN of 200 could serve as a rough guide.

\subsection{Determining the optimal query signature size}

Above analyses determined XSum as the optimal method and tuned the parameter of topN to 200. Next, we intended to further discern the optimal gene number in query signatures. Considering that $\operatorname{Sig}_{\text {gastro }}(\mathrm{n}=44)$ and $\operatorname{Sig}_{\mathrm{NC}}(\mathrm{n}=73)$ had fixed and relatively small signature sizes, we only utilized signatures generated from LIRI and GSE54236 cohorts for subsequent investigation. We adopted two complementary approaches: (i) iterative fold change-based and (ii) random sampling-based approaches, to obtain query signatures with different sizes (Fig. 3d). The iterative fold change-based approach could create a number of signatures with discontinuous sizes through setting iterative threshold values of fold changes. The exact sizes of optimal signatures identified by this approach varied substantially (including 55, 79, 140, and 167). Despite this, similar trends of biphasic pattern with inflection points at around 100 under different conditions could still be observed (Fig. 3e and Additional file 2: Figure S6a). The approach based on random sampling was adopted as a complement. The results showed that as the signature sizes increased, the DR-AUC/DR-ES values also increased/decreased and eventually converged when the signature size was more than 100 (Fig. 3f and Additional file 2: Figure S6b). Based on these observations, we 
297 considered that the signature size of 100 could be selected as a good compromise.

298 This conclusion remained valid in the conditions when other topN values were

299 applied, such as 100 and 400 (results not shown).

300

\subsection{Investigating the optimal clinical phenotype underlying query signature}

302

303

304

305

306

307

308

309

310

311

312

313

314

315

316

317

318

319

320

321

322

323

324

325

Many previous studies chose to compare normal versus diseased states to define disease signatures. However, signatures derived from other clinical phenotypes, such as prognosis and metastasis, could also be used to query LINCS. Aiming to figure out whether this factor could also affect the performance of drug retrieval, we designed a forward and a backward strategy respectively (Additional file 2: Figure S7a). The application of forward strategy was based on two types of signatures, general signatures (representing discordant expression pattern between normal and tumor tissues) and prognostic signatures (associated with survival outcomes). Through adopting above random sampling-based approach, we compared the above two signature phenotypes across varied signature sizes. Unfortunately, the results under different datasets and benchmarking standards were highly inconsistent. This strategy, consequently, failed to provide a definitive conclusion (Additional file 2: Figure S8 a and $b)$.

Opposed to the forward strategy, backward strategy started from creating a collection of 10,000 random signatures, followed by identification of the optimal signature for clinical implication evaluation. Specifically, the optimal random signature was determined according to both benchmarking standards, and exploring the clinical values of this signature might, to some extent, reveal some necessary features possessed by a 'good' query signature (Additional file 2: Figure S7b). A comprehensive clinical evaluation on the optimal signature was carried out based on five RNAseq and five microarray clinical cohorts from three perspectives. First, the ability of this signature to distinguish tumors from non-tumors was investigated. Briefly, we extracted the first principal components (PC1) of this signature to represent its overall expression pattern. AUC was used here as a reflection of the 
classification capability. The results showed that more than 0.90 of AUC can be obtained in seven out of eight cohorts (87.5\%), indicating that the ability to discern the difference between diseased and normal states might be an indispensable property for achieving good retrieval performance (Additional file 2: Figure S7c). Next, we intended to find out whether the optimal signature should be a prognostic indicator. Cox regression analyses were conducted to investigate the association between the signature expression (PC1) and clinical outcome. As a result, significant prognostic implications (Cox $P<0.05)$ of the optimal signature could be observed in six out of eight cohorts (75\%), suggesting that prognostic significance could also be a necessary characteristic (Additional file 2: Figure S7d). At last, the association between signature expression and other clinical features was explored. Considering that $\mathrm{CHCC}$ and LIHC cohorts held the most abundant clinical information, corresponding analyses were thus conducted on these two cohorts. The results showed that there was a significant correlation between the optimal signature and multiple clinical features, including BCLC stage $(P=0.011)$, tumor thrombus $(P=0.001)$, AFP level $(P=$ 0.022), TNM stage $(P<0.001)$, and histologic grade $(P<0.001)$ (Additional file 2: Figure S7e). According to the above results, we concluded that quality query signature should possess the ability to comprehensively recapitulate the clinical features of corresponding disease, rather than only reflect the disease characteristic from single perspective.

\subsection{Linking above findings to the discovery of new anti-liver cancer therapeutics}

The knowledge acquired from above analyses was then applied to establish a signature characterizing liver cancer initiation and development, so as to identify computationally prioritized compounds with potential therapeutic as well as chemopreventive effects against liver cancer. The generation of this evolution-associated signature was based on the notion that the initiation and progression of liver cancer was a stepwise process with gradually acquired advantageous biological capabilities (Fig. 4a). Therefore, conceptually, antagonizing 
355

356

357

358

359

360

361

362

363

364

365

366

367

368

369

370

371

372

373

374

375

376

377

378

379

380

381

382

383

genes that were most related to these stages could be a potential therapeutic strategy.

Through implementing Random Forests algorithm on GSE89377 cohort, preliminary screening was performed to include stage-associated genes, where genes with greater predictive power were selected for further analysis. This screening yielded a total of 6017 stage-associated genes (23.9\%), of which 309 were landmark genes (Fig. 4b). Next, we conducted weighted gene co-expression network analysis (WGCNA) to obtain co-expressed modules with diverse expression patterns (Additional file 2: Figure S9a). Seven gene modules were discerned by WGCNA analysis (Additional file 2: Figure S9 b and c), and two of them, which we termed the 'ascending' module $(n=1,738)$ and the 'descending' module $(n=350)$ for their greatest relevance to stages and patterns of linear evolution from normal to cancer, were retained for further analyses (Fig. $4 \mathrm{c}$ and d). Biological processes associated with genes in these two modules were investigated. We found that the 'ascending' module was closely associated with proliferation (Fig. 4c), while the 'descending' module was enriched in several different types of processes (Fig. 4d). There were 159 genes in common between these two modules and landmarks. Based on the aforementioned recommend about the optimal query signature size, we sought to further reduce the size of 159 to 100 genes. This procedure was carried out using HCC occurrence-related clinical and molecular data in GSE15654 cohort. In brief, ten thousand random signatures, each containing 100 genes, were generated based on the 159-gene panel. The one which had the most significant association with HCC occurrence was considered as the optimal query signature (Additional file 2: Figure S10 a and b). This analysis yielded a signature comprised 82 ascending genes and 18 descending genes, with cox $P$ value of 0.009, which was then termed as $\operatorname{Sig}_{\text {evo }}$ (Additional file 3: Table S10). The linear evolution pattern of $\mathrm{Sig}_{\mathrm{evo}}$ remained present in training (Additional file 2: Figure S10c) as well as an internal validation cohort (Additional file 2: Figure S10d).

As previously discussed, a 'good' query signature should reflect the clinical features of corresponding disease comprehensively. To evaluate whether $\operatorname{Sig}_{\text {evo }}$ was suitable for querying chemopreventive and therapeutic agents for liver cancer, we 
384 systematically surveyed the association between this signature and the clinical 385 phenotypes of precancerous/cancerous liver lesions using clinical and experimental 386 data from both human and animal datasets. First, based on clinical cohorts of HCC, 387 we demonstrated that $\mathrm{Sig}_{\text {evo }}$ had a remarkable capability for distinguishing between 388 tumors from non-tumors, with a median AUC of 0.972 in all eight cohorts (Fig. 4e). 389 Besides, this signature also held great prognostic power in HCC, as suggested by the 390 results of Cox analyses (Fig. 4f). Next, in view of the crucial role of fibrosis in driving 391 hepatocarcinogenesis, further investigation was performed to validate its relevance to 392 fibrosis-related phenotype. The result suggested that $\operatorname{Sig}_{\text {evo }}$ could also effectively 393 differentiate between mild (S0/S1) and severe (S3/S4) fibrosis, thus demonstrating its 394 relationship with fibrosis progression (Fig. 4g). Additionally, we collected four 395 experimental datasets, including two carbon tetrachloride (CCl4)-treated mouse 396 datasets and two diethylnitrosamine (DEN)-treated rat datasets, to assess the 397 enrichment extent of $\mathrm{Sig}_{\text {evo }}$ in mouse and rat fibrosis models. It could be observed that 398 ascending genes in Sig $_{\text {evo }}$ were significantly enriched in both CCl4-treated 399 (GSE27640) and DEN-treated (GSE19057) liver tissues (Fig. 4 h and i). However, 400 descending genes did not exhibit any significant enrichment pattern in all included 401 datasets, possibly due to the limited gene number (Fig. $4 \mathrm{~h}$ and i). Notably, the 402 expression profiles in GSE63726 were derived from non-parenchymal cell fractions which had abundant hepatic stellate cells (HSCs), and thus the significant enrichment could provide the evidence that this signature might reflect the molecular feature of HSC activation (Fig. 4i). In summary, the $\mathrm{Sig}_{\text {evo }}$ fully complied with the criteria of 'good' query signature and was thus employed for querying LINCS.

407 Subsequently, using the optimal method (XSum) and a compromising parameter 408 (topN $=200$ ), we matched Sig $_{\text {evo }}$ with HepG2-derived compound signatures in LINCS 409 and obtained the similarity scores of each compound (lower scores implied higher 410 reversal potency and greater potential for application). After excluding preclinical 411 agents or agents withdrawn from the market according to the drug annotation 412 achieved from the Drug Repurposing Hub, 793 agents remained, which were then 
413 considered as repositioning candidates (Additional file 3: Table S11) [24].

414 Interestingly, some agents which were previously proved to have chemopreventive 415 effects, including erlotinib [25], caffeine [26], and fasudil [27], dominated relatively 416 high rankings on the list (Fig. 5a). Besides, anti-HCC agents were also found to be 417 enriched significantly in compounds with reversal potency (Fig. 5b). These findings 418 converged together to support the reliability of the prediction results.

\subsection{Characterization of homoharringtonine as anti-liver cancer agent}

421 According to the computational results, homoharringtonine (HHT) (Fig. 5c), a protein 422 synthesis inhibitor targeting RPL3, had the highest reversal potency among 793 423 repositioning candidates [28]. To ensure the reliability of L1000 platform-derived 424 HHT signature, we treated HepG2 cells with the same condition used in LINCS and 425 utilizing RNA sequencing to measure HHT-induced expression changes. Comparison between L1000-based and RNA sequencing-based HHT signatures was made and a good agreement between them could be observed, suggesting that our prediction using L1000-based HHT signature was comparable and reliable (Additional file 2: Figure S11). Further validation was then conducted to investigate the potency of HHT against liver cancer.

As the drug target of HHT, RPL3 were first characterized and delineated for its clinical and biological implications. The comparison of mRNA expression between normal and tumor tissues suggested that RPL3 exhibited a higher expression in tumor tissues (Additional file 2: Figure S12a). The increase of protein expression of RPL3 could also be observed in tumor tissues, as shown by immunohistochemical images 436 from the Human Protein Atlas (Additional file 2: Figure S12b) [29]. Higher expression of RPL3 also indicated a worse prognosis (Additional file 2: Figure S12c). 438 In addition to the clinical implications, RPL3 also had essential biological 439 implications in liver cancer. Leveraging the clustered regularly interspaced short 440 palindromic repeats (CRISPR)-based screening data from Project Achilles, we found 441 that RPL3 was essential for maintaining the survival and growth of all liver cancer 
442 cell lines (Additional file 2: Fig. S12 d and e) [30]. Above results demonstrated the 443 rationality of RPL3 inhibition for treating liver cancer.

$444 \quad$ Subsequently, we analyzed the drug response data from PRISM which measured 445 the HHT activities in a large panel of cell lines [31]. It could be observed that, 446 compared with molecular-targeted agents and non-oncology agents, HHT had a lower 447 distribution of $\mathrm{IC}_{50}$ values across 482 PRISM cell lines (Fig. 5d). Of note, in 15 liver 448 cancer cell lines, HHT has exhibit a powerful anti-cancer capacity with a median IC50 449 value of $0.408 \mu \mathrm{M}$, which was numerically lower than three FDA approved HCC 450 agents (lenvatinib: $0.617 \mu \mathrm{M}$; regorafenib: $2.009 \mu \mathrm{M}$; sorafenib: $3.348 \mu \mathrm{M}$ ) (Fig. 5e). 451 The anti-liver cancer activity of HHT was also corroborated by the long-term cell 452 proliferation assay (Fig. 5f) and IncuCyte cell proliferation assay (Additional file 2: 453 Figure S13). Given that the co-administration of HHT with other approved agents was 454 more likely to have clinical utility, we also interrogated whether HHT had the ability 455 to augment the tumor-killing effect of sorafenib. Three different statistical models were adopted for synergy estimation. The results suggested that HHT could indeed synergize with sorafenib in many conditions, albeit not very remarkable in general (Fig. 5g-h and Additional file 2: Figure S14). Collectively, our results demonstrated the therapeutic potential of HHT, either administrated as monotherapy or as combination with sorafenib.

\subsection{Investigation of the anti-fibrotic effects of homoharringtonine}

463 Liver fibrosis occurs when the liver tissue is repeatedly and continuously injured, which is a crucial risk factor for hepatocarcinogenesis [32]. The activation of hepatic stellate cells (HSCs) is one of the key steps in fibrosis development [33]. Since we have proved that $\mathrm{Sig}_{\text {evo }}$ was associated with liver fibrosis using clinical and animal-derived data, it could be postulated that HHT, a compound with the ability to reverse $\mathrm{Sig}_{\mathrm{evo}}$, might also be able to inhibit the activation of HSCs and thereby alleviate liver fibrosis. To test this hypothesis, the anti-fibrotic effects of 
471 We first treated LX-2 cells with vehicle or HHT $(1 \mu \mathrm{M}$ and $5 \mu \mathrm{M})$ for 6 hours. Then, 472 RNA sequencing was conducted to quantify the mRNA expression level of 473 vehicle-treated or HHT-treated LX-2 cells (Additional file 2: Table S12). 474 HHT-induced expression changes were obtained based on the resultant data. Nine 475 well-acknowledged fibrotic genes from previous publications were collected for 476 subsequent analysis, the high expression level of which represented the activation 477 status of HSCs. Apparently, the mRNA expression of almost all the fibrosis-associated 478 genes were downregulated after HHT treatments (Fig. 6a and Additional file 2: Figure 479 S15). The downregulated tendency of two most critical genes which encoded collagen 480 I and $\alpha$-SMA was further corroborated by the quantitative real-time PCR (Fig. 6b). 481 Additionally, the protein-level expression of collagen I and $\alpha$-SMA was also detected 482 using western blot and immunofluorescence. The results showed that HHT could 483 inhibit the protein expression of collagen I and $\alpha$-SMA as well (Fig. $6 \mathrm{c}$ and d). 484 Overall, the ability of suppressing HSC activation markers of HHT indicated it had 485 potential anti-fibrotic activity and thus may hold promise for preventing 486 hepatocarcinogenesis.

487

\section{Discussion}

In recent years, the explosive growth of pharmacogenomic data enables the

490 development of the new frontier of research named computational drug discovery and 491 repositioning, leading to many remarkable findings of novel therapeutics [34]. Owing 492 to the success of CMap and LINCS projects which profiled numerous 493 compound-induced expression data [4-5], 'signature reversion'-based computational 494 drug discovery approach has been extensively adopted [7-10]. However, lack of 495 suitable benchmarking standards for evaluating drug repositioning performance limits 496 further improvement of this approach. Although some studies proposed that the 497 benchmarks assessing drug-drug similarity, such as the Anatomical Therapeutic 498 Chemical system (ATC fourth level codes), could be taken as alternative standards to 499 indirectly determine the optimal methodologies and parameters of computational 
500 repositioning where gold standards for benchmarking were more complicated [22-23],

501 considering the great difference between these two situations, developing specifically

502 tailored benchmarking standards for assessing disease-drug similarity would be more

503 desirable [21b]. In this study, we proposed two novel benchmarking standards,

504 AUC-based standard and KS statistic-based standard. According to the

505 aforementioned observations, despite being mutually independent, there still existed

506 good agreement of the evaluation results between these two standards, demonstrating

507 the rationality and robustness of the novel standards.

508 These two standards enable the establishment of a standardized procedure for 509 performing more effective signature-based computational repositioning. We first 510 suggested that using reference signatures from one of the most relevant cell lines with 511 the disease of interest instead of from a non-touchstone cell line or aggregation-based 512 consensus results was a preferable option to exploit LINCS data, in view of a high 513 degree of cell-type specific effects of perturbations. Next, XSum was identified as an 514 optimal method for matching compound and disease signatures. Notably, a prior study 515 which made a comparison of drug retrieval performance between XSum, XCos, and 516 KS methods using a totally different benchmarking standard from ours also come to 517 the same conclusion [21b]. This consistency not only further demonstrated the 518 reliability of the two newly developed benchmarking standards, but also could support 519 our identification of using XSum as the optimal method. Furthermore, we also 520 uncovered the parameter choice (topN $=200$ ) of XSum, which lacked guidance 521 previously.

522 Within the computational repositioning framework, most of current investigations 523 and methodological developments were focused on reference signatures and signature 524 matching methods. Nevertheless, relatively limited efforts have been made to 525 standardize the procedures for generating quality query signatures [35]. In this study, 526 two potential factors, signature phenotypes and the signature size that might be 527 associated with the retrieval performance of query signatures, were systematically 528 analyzed. First, we intended to figure out how many genes in the query signatures 
529 could best characterize a disease state. Through adopting two independent approaches, 530 an appropriate query signature size of 100 was determined. However, some prior 531 studies considered a reduced number of 50 as the optimal length of query signatures $532[10 \mathrm{~b}, 36]$. It is reasonable to speculate that the utility of different signature matching 533 methods (XSum in this study and KS-based methods in other studies) and also the 534 different benchmarking standards may be responsible for the discrepancy. Next, we 535 sought to determine the influences brought by the clinical phenotypes of query 536 signatures, so as to identify the necessary properties a 'good' signature needed to 537 possess. Interestingly, the results suggested that a quality query signature should hold 538 the ability to draw a comprehensive picture of clinical features of corresponding 539 disease. This finding seemed to be reasonable since disease was highly likely to be 540 under-represented when creating molecular signature from only one perspective of 541 this disease.

542 Based on these findings, we summarized the best practice approach for 543 LINCS-based computational drug repositioning. The application of this approach to 544 liver cancer was then carried out. An evolution-associated query signature related to 545 the development and progression of liver cancer was constructed for drug retrieval. 546 Following the best practice approach, HHT was identified as the candidate agent for 547 its highest reversal potency. Since the query signature ( $\operatorname{Sig}_{\text {evo }}$ ) possessed the liver 548 cancer initiation and development-associated properties simultaneously, we 549 considered that HHT might have both chemotherapeutic and chemopreventive effects 550 for liver cancer accordingly. For validating the chemotherapeutic effect of HHT, drug 551 sensitivity data from PRISM was analyzed and cell proliferation assays were 552 conducted. The remarkable tumor-killing activity of HHT suggested it could be novel 553 therapeutics for liver cancer. For validating the chemopreventive effect of HHT, an 554 indirect approach focused on investigating the anti-fibrotic effect of HHT was adopted. 555 Our results showed that the expression level of fibrosis-associated genes was 556 dramatically decreased when challenging HSCs with HHT treatments. Inhibition of 557 liver fibrogenesis might prevent the progression of cirrhosis and thereby suppress 
558 HCC tumorigenesis [25]. Therefore, we supposed that HHT had the potential to be 559 taken as chemopreventive agents for liver cancer as well. Notably, in view of the grim 560 prognosis and imperfect treatment modalities of liver cancer, prevention of HCC 561 development in patients at high risk of primary malignancy or following curative 562 treatment rather than treating patients at advanced stages with limited health benefit is 563 theoretically the most desirable approach to improve patient prognosis [27, 37]. As 564 HHT has been approved by FDA for the treatment of chronic myelogenous leukemia 565 (CML) and widely used in clinical practice without serious adverse effects, it may 566 provide a new chemopreventive strategy that merits further validation in clinical trials 567 [38].

568 In this study, we have performed the most comprehensive surveys so far about the 569 influencing factors involved in 'signature reversion'-based drug repositioning. Two 570 novel benchmarking standards are proposed, which provide insight into the evaluation 571 of methodologies developed for signature reversion purpose. Moreover, all the 572 findings in this study are verified independently by at least two different approaches, 573 which can eliminate potential bias and guarantee the reliability of the results. 574 Nevertheless, we also recognize several important limitations. First, with our design, 575 our conclusions are conditional and hold only under the conditions of using 576 compound profiles of HepG2 in LINCS as reference signatures. Further investigations 577 using other LINCS data are required to extend current conclusions to other conditions. 578 Second, the parameters recommended by us, including topN of 200 and query 579 signature size of 100, are more or less based on our subjective judgments and should 580 be taken as a rough guide. Although there are sufficient non-quantitative estimates 581 supporting the use of these two parameters, more efforts are still needed to 582 quantitatively and accurately determine the optimal parameters for this computational 583 approach. Third, in this study we only focused on analyzing the data from the project 584 that utilized transcriptomic platforms to measure cell responses during perturbation 585 experiments, and other omics data which are actively being generated by different 586 LINCS centers might also be a good choice for computational drug discovery and 
587 repositioning [39]. Recently, large-scale resources (CPPA) of perturbed protein

588 responses have been generated [40]. Considering that proteins are the components of

589 the basic functional units in biological pathways, investigating the optimal

590 repositioning strategy based on proteomic resources should also have important

591 implication.

592

\section{4. Conclusions}

594 In summary, our findings fill a knowledge gap in the area of LINCS-based 595 computational repositioning. Through exploiting these findings, we also determined a 596 promising anti-liver cancer agent HHT, of which the chemotherapeutic and 597 chemopreventive effects have been validated experimentally. 


\section{5. Experimental Section}

600 5.1.Data collection

601 LINCS data were downloaded from Gene Expression Omnibus (GEO) database 602 (Phase I: GSE92742, Phase II: GSE70138). Molecular data of clinical and animal 603 datasets were obtained from The Cancer Genome Atlas (TCGA), the Genotype-Tissue 604 Expression (GTEx), GEO and the ArrayExpress databases. Dependency and 605 pharmacogenomic data of cell lines were achieved from the Dependency Map 606 (DepMap) portal. For detailed description, please see the Additional file 1: 607 Supplementary Methods.

608

609 5.2.AUC-based benchmarking standard

610 Since establishing benchmarking standards and datasets across all LINCS cell lines 611 seems to be an enormous undertaking, we mainly focused exclusively on one cell line 612 (HepG2) and one disease (liver cancer) in this study. Two benchmarking standards, 613 namely area under curve (AUC)-based standard and Kolmogorov-Smirnov (KS) 614 statistic-based standard, were generated for evaluating the retrieval performance of 615 disease-compound similarity metrics across different conditions. For establishing 616 AUC-based standard, we collected the drug response data from multiple data sources. 617 Compound $\mathrm{IC}_{50}$ s tested in HepG2 cell line was achieved from ChEMBL (version 27) 618 [41] and Liver Cancer Model Repository (LIMORE) [42] datasets. Compounds 619 among LINCS, ChEMBL, and LIMORE were mapped using compound name 620 followed by manual inspection. Each experiment provided in ChEMBL was also 621 manually checked to ensure the compliance with our requirement. Due to the 622 redundancy of $\mathrm{IC}_{50} \mathrm{~s}$, the median $\mathrm{IC}_{50} \mathrm{~s}$ of certain compound among duplicates was 623 used for representing the activity of this compound. We categorized the compounds 624 into effective $\left(\mathrm{IC}_{50}<10 \mu \mathrm{M}\right)$ and ineffective group (IC50 $\geq 10 \mu \mathrm{M}$ ) according to 625 previous study [10b]. The ability to distinguish between effective and ineffective 626 compounds was taken as a measurement of retrieval performance of different 627 similarity metrics (namely AUC value) [10b]. Notably, some have argued that partial 
628 AUC (limiting false positive rate at 0.1/0.01) might be a more relevant statistic for 629 actual application of drug repositioning [21b, 22]. However, due to the limited size of 630 our benchmarking dataset, adopting partial AUC with even less information could 631 result in loss of statistical precision, and thus the use of the full AUC is preferred in 632 this situation. The statistical significance of AUCs were calculated through 633 performing permutation test. Briefly, we randomly permuted the class labels of the 634 feature vectors and created 10,000 permutations to form a distribution of 'random' 635 AUCs. Then, the $P$ value was determined according to the fraction of 'random' AUCs 636 greater than or equal to the observed AUC [21b]. For distinguishing, AUC used for 637 evaluating drug retrieval performance was renamed as drug retrieval-associated AUC 638 (DR-AUC), the higher values of which indicate better performance.

639

$640 \quad$ 5.3.KS statistic-based benchmarking standard

641 To avoid confusion, it should be noted that KS-based method was also used for 642 calculating disease-compound similarity scores, and the specific details were 643 described below. For generating the benchmarking dataset required for the KS 644 statistic-based standard, we systematically surveyed clinical trials involved in HCC 645 treatment and compiled a set of potential HCC agents (clinicaltrials.gov). Preliminary 646 retrieval yielded 1,999 results, and after removing trials failing to fulfil our 647 requirements, we obtained 254 potential therapeutic agents for HCC. The detailed 648 retrieval process was presented in Figure 3A. To minimize potential selection bias, 649 this process was performed independently by two investigators (C.Y. and X.H.). 650 Perturbagen-induced expression profiles of 27 agents among these tested in HepG2 651 cell line are available in LINCS dataset. Based on the assumption that 652 HCC-associated agents are more likely to reverse HCC signature than random agent 653 combinations, the enrichment capabilities of different similarity metrics could be used 654 to assess their repositioning performance. The calculation of enrichment score (ES) of 655 HCC agents was generally identical with that in gene set enrichment analysis [43]. 656 Considering that there was no need accounting for the size of the agents set, we did 
657 not calculate the normalized enrichment score (NES) that might introduce additional

658 randomization. To obtain a nominal $P$ value, we created 10,000 permutations and

659 recomputed the ES for each permutation to form a null distribution. The significance

$660 P$ of the observed ES was then determined relative to the null distribution. Notably,

661 ES used in retrieval performance evaluation was renamed as drug retrieval-associated

662 ES (DR-ES), the lower values of which represent better performance.

663

664 5.4.Signature matching methods

665 The retrieval performance of six different matching methods, including eXtreme Sum

666 (XSum) [21b], eXtreme Cosine (XCos) [21b, 22], eXtreme Pearson (XCor) [23],

667 eXtreme Spearman (XSpe) [23], Kolmogorov-Smirnov (KS) test [4], and the Reverse

668 Gene Expression Score (RGES) [10b], was systematically benchmarked. Based on the

669 consideration that small variations in expression changes may be noise without

670 biological significance, the eXtreme methods only utilized top up- and

671 down-regulated genes in compound signatures for similarity score calculation (all

672 remaining genes were assigned the values of zero). By comparison, KS and RGES

673 methods use complete compound profiles as reference signatures. The detailed

674 features and scoring schemes of these methods are described as follows.

675 The XSum method handles the up- and down-regulated genes separately. In brief,

676 the sums of the change values in reference/compound signatures relative to

677 up-regulated query/disease genes $\left(\mathrm{sum}_{\mathrm{up}}\right)$ and down-regulated query/disease genes

$678\left(\right.$ sum $\left._{\text {down }}\right)$ are first calculated. Then, XSum is defined as following: $X S u m=$ sum $_{\text {up }}$

679 sum $_{\text {down }}$. Other three eXtreme methods, including XCos, XCor, and XSpe, take

680 disease signatures as a whole to query compound signatures, and they calculated the

681 correlation between the numeric vectors of disease and compound signatures using

682 cosine similarity, Pearson correlation, and spearman correlation, respectively. Notably,

683 cosine similarity is nearly identical with Pearson correlation except without centering

684 vectors around the mean values. The KS method was adopted by the first CMap study

685 and has been the most widely used method for connecting disease signatures to 
686 compound signatures [4]. Similar to XSum, KS method also needs to sperate disease

687 signatures into two gene sets, up-regulated gene set and down-regulated gene set, and

688 ignores the magnitude of differential expression. Briefly, using complete compound 689 profiles as reference, maximum deviation (MD)-based enrichment scores of 690 up-regulated gene set ( $\left.\mathrm{es}_{\mathrm{up}}\right)$ and down-regulated gene set $\left(\mathrm{es}_{\mathrm{down}}\right)$ are first computed. If $691 \mathrm{es}_{\mathrm{up}}$ and $\mathrm{es}_{\mathrm{down}}$ have the same algebraic sign then KSscore $=0$, otherwise, KSscore $=$ $692 \mathrm{es}_{\text {up }}-\mathrm{es}_{\mathrm{down}}$. The RGES method is a recently proposed modification of the original KS 693 method, which was demonstrated to perform better in drug response prediction than 694 KS method [10b]. In contrast to original KS method, RGES focuses on the reversal 695 relation between the disease and agents, and RGES is defined as $\mathrm{es}_{\mathrm{up}}-\mathrm{es}_{\text {down }}$ 696 regardless of the sign of $\mathrm{es}_{\mathrm{up}}$ and $\mathrm{es}_{\mathrm{down}}$. In addition to the above six methods, there 697 also exist many other methods, such as WSS/sscMap [44], TES [45], ProbCmap [36], 698 NFFinder [46], EMUDRA [23], and so on, for calculating the similarity between 699 disease and compound signatures. However, some of them are not accessible currently 700 and some are developed based on the data of initial CMap dataset (1,309 compounds), 701 and accordingly, we did not include these methods into our analyses.

702

\subsection{Generation of query signatures for performance evaluation}

704 For evaluating retrieval performance of similarity metrics at different conditions, we 705 prepared four HCC-associated gene signatures to query LINCS. Two of them, Siggastro 706 and Sig $_{\mathrm{NC}}$, are achieved from previously published studies [10]. Given that the 707 development of these two signatures were mainly based on LIHC cohort, as a 708 complement, the other two were generated from another RNAseq cohort (LIRI) and a 709 microarray cohort (GSE54236), respectively. The differentially expressed genes in 710 LIRI cohort were computed using edgeR package (version 3.26.5) on raw count data 711 [47]. For microarray data, we used limma package (version 3.40.2) to conduct 712 differential expression analysis on normalized data [48]. The statistically significant 713 differential genes in above analyses were defined as adjusted $P<0.01$ and absolute $714 \log _{2}$ fold change $(\mathrm{FC})>1$. As a result, we obtained a 70 -gene signature $\left(\operatorname{Sig}_{\text {LIRI }}\right)$ with 
71548 up- and 22 down-regulated genes from LIRI and a 28 -gene signature ( $\left.\operatorname{Sig}_{\text {GSE54236 }}\right)$

716 with 22 up- and 6 down-regulated genes from GSE54236 respectively, which could

717 represent discordant expression pattern of HCC. Considering that the gene numbers in

718 signatures created through differential expression analysis were much less than that in

719 prognostic signatures (see section below), to make comparison of retrieval

720 performance between these two signature phenotypes without being subject to the

721 signature size, we relaxed the significance threshold of differential genes to $P<0.01$

722 and $\log _{2} \mathrm{FC}>0.5$, and built two increased signatures which included 125 genes (LIRI)

723 and 116 genes (GSE54236) respectively. These two increased signatures were also

724 used to explore the potential influences of signature size.

725

\subsection{Construction of size-diversified query signatures}

727 We adopted two independent approaches to explore whether the differences of 728 query/disease signature size could affect subsequent drug retrieval. The first approach 729 was based on iterating the threshold of fold change values, ranging from $0.1 /-0.1$ to 730 the maximum/minimum with a increment/decrement of 0.05 , which could obtain a 731 number of query signatures with a diversity of signature size (duplicates were 732 removed). As for the second approach, above two increased signatures, 125-gene 733 signature from LIRI and 116-gene signature from GSE54236, were taken as the basis 734 for generating smaller-size testing signatures. Briefly, we randomly extracted testing 735 signatures from complete signatures, with the size ranging from the minimum of five to the maximum of 124 or 115 . To avoid bias, this process was repeated 1,000 times to generate 1,000 testing signatures for each signature size.

\subsection{Construction of query signatures representing different clinical phenotypes}

740 To investigate whether the clinical phenotype of signature was potential factor

741 affecting the retrieval performance, we developed two strategies, a forward strategy

742 starting from generation of signatures with distinguishing clinical phenotypes to the

743 evaluation of retrieval performance and a backward strategy starting from obtaining 
744 signature with the best performance to the comprehensive investigations of its clinical

745 implication. For the first strategy, to compare with general HCC signature

746 representing discordant expression pattern, two prognostic signatures based on LIRI

747 and GSE54236 cohorts were constructed. We integrated survival data with expression

748 data and performed Cox proportional hazards regression to assess association between

749 overall survival and gene expression. The statistically significant prognostic genes

750 were defined as $P<0.005$. A 133-gene prognostic signature with 117 poor- and 16

751 good-outcome genes was generated based on LIRI, while analysis on GSE54236

752 resulted in a 107-gene prognostic signature with 79 poor- and 28 good-outcome genes.

753 Comparisons of drug retrieval performance between these two types of signatures

754 were carried out subsequently. For the second strategy, taking 978 landmark genes as

755 a basis, simple random sampling without replacement (SRSWOR) was performed to

756 extract genes from landmarks for forming candidate signatures. The size of

757 randomized signatures was set at 100 and the process of random sampling was

758 repeated 10,000 times to obtain a collection of 10,000 randomized signatures. The

759 DR-AUC and DR-ES values were then calculated for each signature, and the optimal

760 one was defined as the signature with the minimum of DR-AUC multiplying DR-ES.

761

$762 \quad$ 5.8.Generation of evolution-associated query signature

763 To find compounds with potential in the prevention and treatment of liver cancer, we

764 developed a hepatocarcinogenesis and progression-associated signature. GSE89377

765 cohort was utilized to build this signature while GSE6764 cohort was taken for

766 external validation. To identify genes associated with developmental stages, we

767 constructed Random Forests (RF) model taking stages as dependent variable. Variable

768 importance was assessed with the mean decrease accuracy (MDA) measures for

769 individual factors in RF model. Variables with positive MDA values are of high

770 importance in predicting stages. In other words, these variables are more likely to be

771 related with liver cancer development and progression (negative MDA values can be

772 regarded as equivalent to zero importance with no predictive power). The RF analysis 
773 was independently repeated 1,000 times with 1,000 trees growing each time, and

774 genes with positive MDA values incorporated in more than 200 iterations were kept

775 for subsequent analyses.

776 We next performed weighted gene co-expression network analysis (WGCNA) to 777 assign resultant genes into modules according to expression similarity, and 778 simultaneously, to recognize the trajectories of gene expression during liver cancer 779 development [49]. First, an appropriate soft threshold was estimated by using the 780 pickSoftThreshold function in WGCNA package. Then, we constructed WGCNA 781 network and detected gene expression modules using blockwiseModules function with 782 a minimum module gene number of 50, soft thresholded power of 12 , and a 783 dendrogram cut height of 0.3 . Genes without assignment to specific modules were 784 assigned the color of grey. Module eigengenes (MEs) representing the first principal 785 components (PC1) of each module were returned, and the module-trait relationship 786 (MTR) analysis was conducted by calculating the correlation between MEs and 787 developmental stages. The expression trend of each module across seven stages of 788 HCC development was visualized through using mean PC1 values of samples in each 789 stage to generate trend curves. According to the correlation coefficient of MTR 790 analysis and the visualized expression trend of each module, two modules exhibiting 791 the highest positive/negative correlation with developmental stages as well as

792 showing gradually increasing or decreasing expression trends were selected for their 793 potential functional roles during HCC initiation and development. Subsequently, to explore the biological processes associated with genes in these two modules, we conducted hypergeometric test based on the hallmark gene sets (h.all.v7.0.symbols)

796 downloaded from the Molecular Signatures Database (MSigDB) using enricher 797 function in clusterProfiler package [50]. The $P$ values from the hypergeometric tests 798 were adjusted for multiple comparison testing and an adjusted $P$ value less than 0.05 799 was considered significantly enriched.

800 Genes in these two modules were mapped to the 978 landmark genes, resulting in 801 a 159-gene panel (134 genes in ascending module and 25 genes in descending 
802 module). According to the findings described in Results section, we decided to further

803 narrow down this panel to create a query signature with the size of 100 genes. The

804 molecular and clinical data in GSE15654 were utilized to determine the association

805 between the expression patterns of signatures and the occurrence of HCC. Briefly, we

806 first performed SRSWOR to extract a subset of 100 genes from the 159-gene panel,

807 repeated 10,000 times. The processes of random sampling were conducted separately

808 for ascending and descending genes, to ensure a minimum descending gene number of

809 two in the randomized signatures. As a result, 10,000 randomized signatures with 100

810 genes per signature were generated. Next, PC1 values of all randomized signatures

811 were extracted based on expression data from GSE15654 to represent the overall

812 expression patterns of these signatures, and the follow-up data using HCC occurrence

813 as endpoint was then integrated with above expression pattern data for subsequent

814 Cox proportional hazards regression $(\mathrm{COXPH})$. The signature which had the

815 minimum $P$ value across the 10,000 COXPH analyses was considered as the optimal

816 signature and retained for querying LINCS. The expression trend of this signature was

817 further validated GSE6764 cohort.

818

\section{5.9.Experimental methods}

820 All experimental methods, including cell proliferation assays, quantitative real-time

821 PCR, western blotting analysis, immunofluorescence, RNA sequencing, etc., were

822 described in the Additional file 1: Supplementary Methods.

823

$824 \quad 5.10$. Statistical analysis

825 All the computational analyses and graphical visualization were performed in $\mathrm{R}$ 826 software, version 3.6.0 (https://cran.r-project.org/). Unless stated otherwise, 827 correlation between two continuous variables was measured by Spearman's 828 rank-order correlation, and pairwise comparisons were conducted using 829 Kruskal-Wallis and Wilcoxon sum rank tests. ROC curves and AUC values were 830 visualized and calculated using the pROC package[51]. The hazard ratio (HR) was 
831 estimated using Cox regression model in survival $\mathrm{R}$ package. Cumulative hazard 832 curve was carried out using jskm package and the log-rank test was used to determine 833 the statistical significance of differences. A two-tailed $P<0.05$ was considered 834 significant unless indicated otherwise.

835

836

Additional files

837 Additional file 1: Supplementary methods and Supplementary discussion. (DOCX 113 $838 \mathrm{~kb})$

839 Additional file 2: Supplementary figures 1-15. (PDF $4333 \mathrm{~kb}$ )

840 Additional file 3: Supplementary tables 1-12. (XLS $2279 \mathrm{~kb}$ )

841

842 Availability of data and materials

843 Sequencing data necessary for the analysis are available within Supplementary Data.

844 The rest of the data supporting the findings of this study are available from the 845 corresponding author upon reasonable request. The codes are available at 846 https://github.com/YangJAT/Best practices for LINCS.

847

848 Acknowledgements

849 This work was financially supported by the National Natural Science Foundation of 850 China (81972208), Shanghai Natural Science Foundation (19ZR1452700).

851

\section{Conflict of interest}

853 The authors declare no conflict of interest.

854

855 Authors' Contributions

856 C.Y. and M.C. designed the study and performed the computational analyses. S.W. 857 and X.H. and R.Q. performed the in vitro validation experiments. C.Y., J.W. and Z.L. 858 wrote the manuscript. Q.W. provided guidance on manuscript preparation. H.W., H.H. 859 and C.W. supervised the study. 
bioRxiv preprint doi: https://doi.org/10.1101/2021.06.29.450305; this version posted June 30, 2021. The copyright holder for this preprint (which was not certified by peer review) is the author/funder. All rights reserved. No reuse allowed without permission.

860

861 
862

863 [1] J. W. Scannell, A. Blanckley, H. Boldon, B. Warrington, Nat. Rev. Drug Discov. 2012, 11

864 (3), 191, https://doi.org/10.1038/nrd3681.

865

866

867

868

869

870

871

872

873

874

875

876

877

878

879

880

881

882

883

\section{References}

[2] a) T. T. Ashburn, K. B. Thor, Nat. Rev. Drug Discov. 2004, 3 (8), 673, https://doi.org/10.1038/nrd1468; b) Z. Liu, H. Fang, K. Reagan, X. Xu, D. L. Mendrick, W. Slikker, Jr., W. Tong, Drug Discov. Today 2013, 18 (3-4), 110, https://doi.org/10.1016/j.drudis.2012.08.005.

[3] J. Li, S. Zheng, B. Chen, A. J. Butte, S. J. Swamidass, Z. Lu, Brief. Bioinform. 2016, 17(1),

\section{2, https://doi.org/10.1093/bib/bbv020.}

[4] J. Lamb, E. D. Crawford, D. Peck, J. W. Modell, I. C. Blat, M. J. Wrobel, J. Lerner, J. P. Brunet, A. Subramanian, K. N. Ross, M. Reich, H. Hieronymus, G. Wei, S. A. Armstrong, S. J. Haggarty, P. A. Clemons, R. Wei, S. A. Carr, E. S. Lander, T. R. Golub, Science 2006, 313 (5795), 1929, https://doi.org/10.1126/science.1132939.

[5] A. Subramanian, R. Narayan, S. M. Corsello, D. D. Peck, T. E. Natoli, X. Lu, J. Gould, J. F. Davis, A. A. Tubelli, J. K. Asiedu, D. L. Lahr, J. E. Hirschman, Z. Liu, M. Donahue, B. Julian, M. Khan, D. Wadden, I. C. Smith, D. Lam, A. Liberzon, C. Toder, M. Bagul, M. Orzechowski, O. M. Enache, F. Piccioni, S. A. Johnson, N. J. Lyons, A. H. Berger, A. F. Shamji, A. N. Brooks, A. Vrcic, C. Flynn, J. Rosains, D. Y. Takeda, R. Hu, D. Davison, J. Lamb, K. Ardlie, L. Hogstrom, P. Greenside, N. S. Gray, P. A. Clemons, S. Silver, X. Wu, W. N. Zhao, W. Read-Button, X. Wu, S. J. Haggarty, L. V. Ronco, J. S. Boehm, S. L. Schreiber, J. G. Doench, J. A. Bittker, D. E.

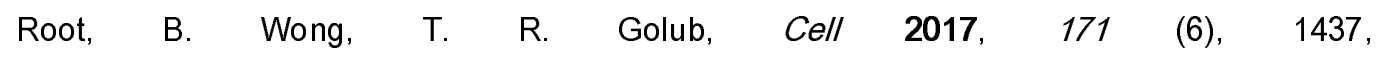
https://doi.org/10.1016/j.cell.2017.10.049. 
884 [6] A. Musa, L. S. Ghoraie, S. D. Zhang, G. Glazko, O. Yli-Harja, M. Dehmer, B. Haibe-Kains,

885 F. Emmert-Streib, Brief. Bioinform. 2018, 19 (3), 506, https://doi.org/10.1093/bib/bbw112.

886 [7] G. Wei, D. Twomey, J. Lamb, K. Schlis, J. Agarwal, R. W. Stam, J. T. Opferman, S. E.

887 Sallan, M. L. den Boer, R. Pieters, T. R. Golub, S. A. Armstrong, Cancer Cel/2006, 10(4), 331, 888 https://doi.org/10.1016/j.ccr.2006.09.006.

889 [8] J. T. Dudley, M. Sirota, M. Shenoy, R. K. Pai, S. Roedder, A. P. Chiang, A. A. Morgan, M. 890 M. Sarwal, P. J. Pasricha, A. J. Butte, Sci. Transl. Med. 2011, 3 (96), 96ra76, 891 https://doi.org/10.1126/scitranslmed.3002648.

892 [9] V. van Noort, S. Schölch, M. Iskar, G. Zeller, K. Ostertag, C. Schweitzer, K. Werner, J. 893 Weitz, M. Koch, P. Bork, Cancer Res. 2014, 74 (20), 5690, 894 https://doi.org/10.1158/0008-5472.Can-13-3540.

895 [10] a) B. Chen, W. Wei, L. Ma, B. Yang, R. M. Gill, M. S. Chua, A. J. Butte, S. So, 896 Gastroenterology 2017, 152 (8), 2022, https://doi.org/10.1053/j.gastro.2017.02.039; b) B.

897 Chen, L. Ma, H. Paik, M. Sirota, W. Wei, M. S. Chua, S. So, A. J. Butte, Nature 898 communications 2017, 8, 16022, https://doi.org/10.1038/ncomms16022.

899 [11] F. Bray, J. Ferlay, I. Soerjomataram, R. L. Siegel, L. A. Torre, A. Jemal, CA Cancer J. Clin. 900 2018, 68(6), 394, https://doi.org/10.3322/caac.21492.

901 [12] J. M. Llovet, J. Zucman-Rossi, E. Pikarsky, B. Sangro, M. Schwartz, M. Sherman, G. 902 Gores, Nature reviews. Disease primers 2016, 2, 16018, https://doi.org/10.1038/nrdp.2016.18.

903 [13] M. Kudo, R. S. Finn, S. Qin, K. H. Han, K. Ikeda, F. Piscaglia, A. Baron, J. W. Park, G. 904 Han, J. Jassem, J. F. Blanc, A. Vogel, D. Komov, T. R. J. Evans, C. Lopez, C. Dutcus, M. Guo, 905 K. Saito, S. Kraljevic, T. Tamai, M. Ren, A. L. Cheng, Lancet 2018, 391 (10126), 1163, 
906

907

908

909

910

911

912

913

914

915

916

917

918

919

920

921

922

923

924

925

926

927

https://doi.org/10.1016/s0140-6736(18)30207-1.

[14] J. Bruix, S. Qin, P. Merle, A. Granito, Y. H. Huang, G. Bodoky, M. Pracht, O. Yokosuka, O.

Rosmorduc, V. Breder, R. Gerolami, G. Masi, P. J. Ross, T. Song, J. P. Bronowicki, I.

Ollivier-Hourmand, M. Kudo, A. L. Cheng, J. M. Llovet, R. S. Finn, M. A. LeBerre, A.

Baumhauer, G. Meinhardt, G. Han, Lancet 2017, 389 (10064), 56, https://doi.org/10.1016/s0140-6736(16)32453-9.

[15] G. K. Abou-Alfa, T. Meyer, A. L. Cheng, A. B. El-Khoueiry, L. Rimassa, B. Y. Ryoo, I. Cicin, P. Merle, Y. Chen, J. W. Park, J. F. Blanc, L. Bolondi, H. J. Klümpen, S. L. Chan, V. Zagonel, T. Pressiani, M. H. Ryu, A. P. Venook, C. Hessel, A. E. Borgman-Hagey, G. Schwab, R. K. Kelley, N. Engl. J. Med. 2018, 379(1), 54, https://doi.org/10.1056/NEJMoa1717002.

[16] A. X. Zhu, Y. K. Kang, C. J. Yen, R. S. Finn, P. R. Galle, J. M. Llovet, E. Assenat, G. Brandi, M. Pracht, H. Y. Lim, K. M. Rau, K. Motomura, I. Ohno, P. Merle, B. Daniele, D. B. Shin, G. Gerken, C. Borg, J. B. Hiriart, T. Okusaka, M. Morimoto, Y. Hsu, P. B. Abada, M. Kudo, Lancet Oncol. 2019, 20(2), 282, https://doi.org/10.1016/s1470-2045(18)30937-9.

[17] R. S. Finn, B. Y. Ryoo, P. Merle, M. Kudo, M. Bouattour, H. Y. Lim, V. Breder, J. Edeline, Y. Chao, S. Ogasawara, T. Yau, M. Garrido, S. L. Chan, J. Knox, B. Daniele, S. W. Ebbinghaus, E. Chen, A. B. Siegel, A. X. Zhu, A. L. Cheng, J. Clin. Oncol. 2020, 38 (3), 193, https://doi.org/10.1200/jco.19.01307.

[18] A. B. El-Khoueiry, B. Sangro, T. Yau, T. S. Crocenzi, M. Kudo, C. Hsu, T. Y. Kim, S. P. Choo, J. Trojan, T. H. R. Welling, T. Meyer, Y. K. Kang, W. Yeo, A. Chopra, J. Anderson, C. Dela Cruz, L. Lang, J. Neely, H. Tang, H. B. Dastani, I. Melero, Lancet 2017, 389 (10088), 2492, https://doi.org/10.1016/s0140-6736(17)31046-2. 
928

929

930

931

932

933

934

935

936

937

938

939

940

941

942

943

944

945

946

947

948

949

[19] R. S. Finn, S. Qin, M. Ikeda, P. R. Galle, M. Ducreux, T. Y. Kim, M. Kudo, V. Breder, P. Merle, A. O. Kaseb, D. Li, W. Verret, D. Z. Xu, S. Hernandez, J. Liu, C. Huang, S. Mulla, Y. Wang, H. Y. Lim, A. X. Zhu, A. L. Cheng, N. Engl. J. Med. 2020, 382 (20), 1894, https://doi.org/10.1056/NEJMoa1915745.

[20] a) M. Niepel, M. Hafner, Q. Duan, Z. Wang, E. O. Paull, M. Chung, X. Lu, J. M. Stuart, T. R. Golub, A. Subramanian, A. Ma'ayan, P. K. Sorger, Nature communications 2017, 8 (1), 1186, https://doi.org/10.1038/s41467-017-01383-w; b) Z. Wang, A. Lachmann, A. B. Keenan, A. Ma'ayan, Bioinformatics 2018, 34 (12), 2150, https://doi.org/10.1093/bioinformatics/bty060. [21] a) A. Wagner, N. Cohen, T. Kelder, U. Amit, E. Liebman, D. M. Steinberg, M. Radonjic, E. Ruppin, Mol. Syst. Biol. 2015, 11 (3), 791, https://doi.org/10.15252/msb.20145486; b) J. Cheng, L. Yang, V. Kumar, P. Agarwal, Genome Med. 2014, 6 (12), 540, https://doi.org/10.1186/s13073-014-0095-1.

[22] J. Cheng, Q. Xie, V. Kumar, M. Hurle, J. M. Freudenberg, L. Yang, P. Agarwal, Pac. Symp. Biocomput. 2013, 5.

[23] X. Zhou, M. Wang, I. Katsyv, H. Irie, B. Zhang, Bioinformatics 2018, 34 (18), 3151, https://doi.org/10.1093/bioinformatics/bty325.

[24] S. M. Corsello, J. A. Bittker, Z. Liu, J. Gould, P. McCarren, J. E. Hirschman, S. E. Johnston, A. Vrcic, B. Wong, M. Khan, J. Asiedu, R. Narayan, C. C. Mader, A. Subramanian, T. R. Golub, Nat. Med. 2017, 23 (4), 405, https://doi.org/10.1038/nm.4306.

[25] B. C. Fuchs, Y. Hoshida, T. Fujii, L. Wei, S. Yamada, G. Y. Lauwers, C. M. McGinn, D. K. DePeralta, X. Chen, T. Kuroda, M. Lanuti, A. D. Schmitt, S. Gupta, A. Crenshaw, R. Onofrio, B. Taylor, W. Winckler, N. Bardeesy, P. Caravan, T. R. Golub, K. K. Tanabe, Hepatology 2014, 
950

951

952

953

954

955

956

957

958

959

960

961

962

963

964

965

966

967

968

969

970

971

59 (4), 1577, https://doi.org/10.1002/hep.26898.

[26] Y. Hoshida, B. C. Fuchs, K. K. Tanabe, Curr. Cancer Drug Targets 2012, 12 (9), 1129.

[27] S. Nakagawa, L. Wei, W. M. Song, T. Higashi, S. Ghoshal, R. S. Kim, C. B. Bian, S. Yamada, X. Sun, A. Venkatesh, N. Goossens, G. Bain, G. Y. Lauwers, A. P. Koh, M. El-Abtah, N. B. Ahmad, H. Hoshida, D. J. Erstad, G. Gunasekaran, Y. Lee, M. L. Yu, W. L. Chuang, C. Y. Dai, M. Kobayashi, H. Kumada, T. Beppu, H. Baba, M. Mahajan, V. D. Nair, M. Lanuti, A. Villanueva, A. Sangiovanni, M. lavarone, M. Colombo, J. M. Llovet, A. Subramanian, A. M. Tager, S. L. Friedman, T. F. Baumert, M. E. Schwarz, R. T. Chung, K. K. Tanabe, B. Zhang, B. C. Fuchs, Y. Hoshida, Cancer Cell 2016, $30 \quad$ (6), 879, https://doi.org/10.1016/j.ccell.2016.11.004.

[28] R. M. Tujebajeva, D. M. Graifer, G. G. Karpova, N. A. Ajtkhozhina, FEBS Lett. 1989, 257 (2), 254, https://doi.org/10.1016/0014-5793(89)81546-7.

[29] M. Uhlén, L. Fagerberg, B. M. Hallström, C. Lindskog, P. Oksvold, A. Mardinoglu, Å. Sivertsson, C. Kampf, E. Sjöstedt, A. Asplund, I. Olsson, K. Edlund, E. Lundberg, S. Navani, C. A. Szigyarto, J. Odeberg, D. Djureinovic, J. O. Takanen, S. Hober, T. Alm, P. H. Edqvist, H. Berling, H. Tegel, J. Mulder, J. Rockberg, P. Nilsson, J. M. Schwenk, M. Hamsten, K. von Feilitzen, M. Forsberg, L. Persson, F. Johansson, M. Zwahlen, G. von Heijne, J. Nielsen, F. Pontén, Science 2015, 347(6220), 1260419, https://doi.org/10.1126/science.1260419.

[30] R. M. Meyers, J. G. Bryan, J. M. McFarland, B. A. Weir, A. E. Sizemore, H. Xu, N. V. Dharia, P. G. Montgomery, G. S. Cowley, S. Pantel, A. Goodale, Y. Lee, L. D. Ali, G. Jiang, R. Lubonja, W. F. Harrington, M. Strickland, T. Wu, D. C. Hawes, V. A. Zhivich, M. R. Wyatt, Z. Kalani, J. J. Chang, M. Okamoto, K. Stegmaier, T. R. Golub, J. S. Boehm, F. Vazquez, D. E. 
972 Root, W. C. Hahn, A. Tsherniak, Nat. Genet. 2017, 49 (12), 1779, 973 https://doi.org/10.1038/ng.3984.

974 [31] S. M. Corsello, R. T. Nagari, R. D. Spangler, J. Rossen, M. Kocak, J. G. Bryan, R. 975 Humeidi, D. Peck, X. Wu, A. A. Tang, V. M. Wang, S. A. Bender, E. Lemire, R. Narayan, P. 976 Montgomery, U. Ben-David, C. W. Garvie, Y. Chen, M. G. Rees, N. J. Lyons, J. M. McFarland, 977 B. T. Wong, L. Wang, N. Dumont, P. J. O'Hearn, E. Stefan, J. G. Doench, C. N. Harrington, H. 978 Greulich, M. Meyerson, F. Vazquez, A. Subramanian, J. A. Roth, J. A. Bittker, J. S. Boehm, C. 979 C. Mader, A. Tsherniak, T. R. Golub, Nature cancer 2020, 1 (2), 235, 980 https://doi.org/10.1038/s43018-019-0018-6.

981 [32] J. M. O'Rourke, V. M. Sagar, T. Shah, S. Shetty, World J. Gastroenterol. 2018, 24 (39), 982 4436, https://doi.org/10.3748/wjg.v24.i39.4436.

983 [33] W. C. Zhou, Q. B. Zhang, L. Qiao, World J. Gastroenterol. 2014, 20 (23), 7312, 984 https://doi.org/10.3748/wjg.v20.i23.7312.

985 [34] a) V. Stathias, A. M. Jermakowicz, M. E. Maloof, M. Forlin, W. Walters, R. K. Suter, M. A. 986 Durante, S. L. Williams, J. W. Harbour, C. H. Volmar, N. J. Lyons, C. Wahlestedt, R. M. 987 Graham, M. E. Ivan, R. J. Komotar, J. N. Sarkaria, A. Subramanian, T. R. Golub, S. C. Schürer, 988 N. G. Ayad, Nature communications 2018, $9 \quad$ (1), 5315 , 989 https://doi.org/10.1038/s41467-018-07659-z; b) J. Kong, H. Lee, D. Kim, S. K. Han, D. Ha, K. 990 Shin, S. Kim, Nature communications 2020, $11 \quad$ (1), 5485, 991 https://doi.org/10.1038/s41467-020-19313-8; c) C. Yang, J. Chen, Y. Li, X. Huang, Z. Liu, J. 992 Wang, H. Jiang, W. Qin, Y. Lv, H. Wang, C. Wang, Brief Bioinform 2020, 993 https://doi.org/10.1093/bib/bbaa295. 
994 [35] a) Q. Wen, P. O'Reilly, P. D. Dunne, M. Lawler, S. Van Schaeybroeck, M. Salto-Tellez, P.

995 Hamilton, S. D. Zhang, BMC Syst. Biol. 2015, 9 Suppl 5 (Suppl 5), S4,

996 https://doi.org/10.1186/1752-0509-9-s5-s4; b) Q. Wen, C. S. Kim, P. W. Hamilton, S. D. Zhang,

997 BMC Bioinformatics 2016, 17(1), 211, https://doi.org/10.1186/s12859-016-1066-x; c) J. Chan,

998 X. Wang, J. A. Turner, N. E. Baldwin, J. Gu, Bioinformatics 2019, 35 (16), 2818,

999 https://doi.org/10.1093/bioinformatics/btz006.

1000 [36] J. A. Parkkinen, S. Kaski, BMC Bioinformatics 2014, $15, \quad 113$, 1001 https://doi.org/10.1186/1471-2105-15-113.

1002 [37] N. Fujiwara, S. L. Friedman, N. Goossens, Y. Hoshida, J. Hepatol. 2018, 68 (3), 526, $1003 \quad$ https://doi.org/10.1016/j.jhep.2017.09.016.

1004 [38] H. M. Kantarjian, S. O'Brien, J. Cortes, Clin. Lymphoma Myeloma Leuk. 2013, 13(5), 530, 1005 https://doi.org/10.1016/j.clml.2013.03.017.

1006 [39] a) A. B. Keenan, S. L. Jenkins, K. M. Jagodnik, S. Koplev, E. He, D. Torre, Z. Wang, A. B. 1007 Dohlman, M. C. Silverstein, A. Lachmann, M. V. Kuleshov, A. Ma'ayan, V. Stathias, R. Terryn, 1008 D. Cooper, M. Forlin, A. Koleti, D. Vidovic, C. Chung, S. C. Schürer, J. Vasiliauskas, M. 1009 Pilarczyk, B. Shamsaei, M. Fazel, Y. Ren, W. Niu, N. A. Clark, S. White, N. Mahi, L. Zhang, M. 1010 Kouril, J. F. Reichard, S. Sivaganesan, M. Medvedovic, J. Meller, R. J. Koch, M. R. Birtwistle, 1011 R. lyengar, E. A. Sobie, E. U. Azeloglu, J. Kaye, J. Osterloh, K. Haston, J. Kalra, S. Finkbiener, 1012 J. Li, P. Milani, M. Adam, R. Escalante-Chong, K. Sachs, A. Lenail, D. Ramamoorthy, E. 1013 Fraenkel, G. Daigle, U. Hussain, A. Coye, J. Rothstein, D. Sareen, L. Ornelas, M. Banuelos, B. 1014 Mandefro, R. Ho, C. N. Svendsen, R. G. Lim, J. Stocksdale, M. S. Casale, T. G. Thompson, J. 1015 Wu, L. M. Thompson, V. Dardov, V. Venkatraman, A. Matlock, J. E. Van Eyk, J. D. Jaffe, M. 
1016

1017

1018

1019

1020

1021

1022

1023

1024

1025

1026

1027

1028

1029

1030

1031

1032

1033

1034

1035

1036

1037

Papanastasiou, A. Subramanian, T. R. Golub, S. D. Erickson, M. Fallahi-Sichani, M. Hafner, N.

S. Gray, J. R. Lin, C. E. Mills, J. L. Muhlich, M. Niepel, C. E. Shamu, E. H. Williams, D. Wrobel,

P. K. Sorger, L. M. Heiser, J. W. Gray, J. E. Korkola, G. B. Mills, M. LaBarge, H. S. Feiler, M. A.

Dane, E. Bucher, M. Nederlof, D. Sudar, S. Gross, D. F. Kilburn, R. Smith, K. Devlin, R.

Margolis, L. Derr, A. Lee, A. Pillai, Cell systems 2018, 6 (1), 13, https://doi.org/10.1016/j.cels.2017.11.001; b) A. Koleti, R. Terryn, V. Stathias, C. Chung, D. J.

Cooper, J. P. Turner, D. Vidovic, M. Forlin, T. T. Kelley, A. D'Urso, B. K. Allen, D. Torre, K. M. Jagodnik, L. Wang, S. L. Jenkins, C. Mader, W. Niu, M. Fazel, N. Mahi, M. Pilarczyk, N. Clark, B. Shamsaei, J. Meller, J. Vasiliauskas, J. Reichard, M. Medvedovic, A. Ma'ayan, A. Pillai, S. C. Schürer, Nucleic Acids Res. 2018, 46 (D1), D558, https://doi.org/10.1093/nar/gkx1063.

[40] W. Zhao, J. Li, M. M. Chen, Y. Luo, Z. Ju, N. K. Nesser, K. Johnson-Camacho, C. T. Boniface, Y. Lawrence, N. T. Pande, M. A. Davies, M. Herlyn, T. Muranen, I. K. Zervantonakis, E. von Euw, A. Schultz, S. V. Kumar, A. Korkut, P. T. Spellman, R. Akbani, D. J. Slamon, J. W. Gray, J. S. Brugge, Y. Lu, G. B. Mills, H. Liang, Cancer Cell 2020, 38 (6), 829, https://doi.org/10.1016/j.ccell.2020.10.008.

[41]D. Mendez, A. Gaulton, A. P. Bento, J. Chambers, M. De Veij, E. Félix, M. P. Magariños, J. F. Mosquera, P. Mutowo, M. Nowotka, M. Gordillo-Marañón, F. Hunter, L. Junco, G. Mugumbate, M. Rodriguez-Lopez, F. Atkinson, N. Bosc, C. J. Radoux, A. Segura-Cabrera, A. Hersey, A. R. Leach, Nucleic Acids Res. 2019, 47 (D1), D930, https://doi.org/10.1093/nar/gky1075.

[42] Z. Qiu, H. Li, Z. Zhang, Z. Zhu, S. He, X. Wang, P. Wang, J. Qin, L. Zhuang, W. Wang, F. Xie, Y. Gu, K. Zou, C. Li, C. Li, C. Wang, J. Cen, X. Chen, Y. Shu, Z. Zhang, L. Sun, L. Min, Y. 
1038

1039

1040

1041

1042

1043

1044

1045

1046

1047

1048

1049

1050

1051

1052

1053

1054

1055

1056

1057

1058

1059

Fu, X. Huang, H. Lv, H. Zhou, Y. Ji, Z. Zhang, Z. Meng, X. Shi, H. Zhang, Y. Li, L. Hui, Cancer Cel/2019, 36 (2), 179, https://doi.org/10.1016/j.ccell.2019.07.001.

[43] A. Subramanian, P. Tamayo, V. K. Mootha, S. Mukherjee, B. L. Ebert, M. A. Gillette, A. Paulovich, S. L. Pomeroy, T. R. Golub, E. S. Lander, J. P. Mesirov, Proc. Natl. Acad. Sci. U. S. A. 2005, 102 (43), 15545, https://doi.org/10.1073/pnas.0506580102.

[44] a) S. D. Zhang, T. W. Gant, BMC Bioinformatics 2008, 9, 258, https://doi.org/10.1186/1471-2105-9-258; b) BMC Bioinformatics 2009, 10, 236, https://doi.org/10.1186/1471-2105-10-236.

[45] F. Iorio, R. Bosotti, E. Scacheri, V. Belcastro, P. Mithbaokar, R. Ferriero, L. Murino, R. Tagliaferri, N. Brunetti-Pierri, A. Isacchi, D. di Bernardo, Proc. Natl. Acad. Sci. U. S. A. 2010, 107 (33), 14621, https://doi.org/10.1073/pnas. 1000138107.

[46] J. Setoain, M. Franch, M. Martínez, D. Tabas-Madrid, C. O. Sorzano, A. Bakker, E. Gonzalez-Couto, J. Elvira, A. Pascual-Montano, Nucleic Acids Res. 2015, 43 (W1), W193, https://doi.org/10.1093/nar/gkv445.

[47] D. J. McCarthy, Y. Chen, G. K. Smyth, Nucleic Acids Res. 2012, 40 (10), 4288, https://doi.org/10.1093/nar/gks042.

[48] M. E. Ritchie, B. Phipson, D. Wu, Y. Hu, C. W. Law, W. Shi, G. K. Smyth, Nucleic Acids Res. 2015, 43(7), e47, https://doi.org/10.1093/nar/gkv007.

[49] P. Langfelder, S. Horvath, BMC Bioinformatics 2008, 9, 559, https://doi.org/10.1186/1471-2105-9-559.

[50] a) A. Liberzon, C. Birger, H. Thorvaldsdóttir, M. Ghandi, J. P. Mesirov, P. Tamayo, Cell systems 2015, 1 (6), 417, https://doi.org/10.1016/j.cels.2015.12.004; b) G. Yu, L. G. Wang, Y. 
bioRxiv preprint doi: https://doi.org/10.1101/2021.06.29.450305; this version posted June 30, 2021. The copyright holder for this preprint (which was not certified by peer review) is the author/funder. All rights reserved. No reuse allowed without permission.

1060 Han, Q. Y. He, OMICS 2012, 16(5), 284, https://doi.org/10.1089/omi.2011.0118.

1061 [51] X. Robin, N. Turck, A. Hainard, N. Tiberti, F. Lisacek, J. C. Sanchez, M. Müller, BMC

1062 Bioinformatics 2011, 12, 77, https://doi.org/10.1186/1471-2105-12-77.

1063

1064

1065 


\section{Figure Legends}

1067 Fig. 1 Overview of LINCS data-driven therapeutic discovery. The working 1068 principle of 'signature reversion'-based computational approach. A disease signature 1069 representing discordant expression pattern needs first to be identified (G1, G2, and G3 1070 stand for up-regulated genes while G4, G5, and G6 stand for down-regulated genes in 1071 disease state). With this signature, pharmacologic perturbation datasets can be queried 1072 to find compounds with the ability to reverse disease expression pattern (suppress 1073 expression of G1, G2, and G3 and induce expression of G4, G5, and G6). After 1074 determining the candidate compounds, experimental and clinical validation are 1075 required to translate computational findings to clinical applications.

Fig. 2 Highly cell-type specific compound-induced expression changes. a Two-dimensional t-SNE projection based on cosine distance between compound signatures. Each dot represents a unique perturbation-induced expression profile, and each color represents one type of cell line. b Schematic diagram displaying the calculation process of compound-level (L2) and MOA-level (L3) similarity scores. c Distribution of compound-level (L2) cosine similarity scores, which ranging from -1 (completely opposite pattern) to 1 (perfectly identical pattern). Three examples are presented (left to right: etodolac, geldanamycin, and doxorubicin). d Illustration of MOA-level (L3) similarities. Only MOAs with more than five compounds included are shown in figure. e A t-SNE projection showing the distribution of compounds (indicated by purple dots) in top ranked five MOAs (including HDAC inhibitors, IKK inhibitors, mTOR inhibitors, CDK inhibitors, and topoisomerase inhibitors). f Schematic diagram displaying the calculation process of cell line pair-level (L4) similarity scores. (g) Correlation between basal expression similarities and perturbed expression similarities (L4) of 36 cell line pairs (nine cell lines in total). Statistical significance and correlation coefficient were determined by ranked-based Spearman correlation. h Schematic view of the calculation of cell line-level (L5) similarity scores (upper) and the presentation of L5 similarity scores of nine cell lines in the 
1095

1096

1097

1098

1099

1100

1101

1102

1103

1104

1105

1106

1107

1108

1109

1110

1111

1112

1113

1114

1115

1116

1117

1118

1119

1120

1121

1122

boxplot (lower).

Fig. 3 Benchmarking different methodologies and parameters to determine the optimal computational strategy. a Diagram summarizing the workflow and the important components involved in the evaluation process of drug retrieval performance of six different signature matching methods. b Retrieval performance of six matching methods evaluated by AUC-based benchmarking standard (left) and KS statistic-based benchmarking standard (right). c Visualization of AUC-based (left) and KS statistic-based (right) performance measurements of XSum method on standardized data for discerning the optimal operating parameter. d Diagram summarizing the workflow and the important components associated with the investigation process of the optimal query signature size. e Relationship between query signature size determined by iterative fold change-based approach and retrieval performance evaluated by AUC-based standard (left) and KS statistic-based standard (right). f Relationship between query signature size determined by random sampling-based approach and retrieval performance evaluated by AUC-based standard (left) and KS statistic-based standard (right). LOESS polynomial regression analysis was performed for curve fitting. Query signature for above analyses was generated based on LIRI cohort, and evaluation results using other query signatures were presented in Supplementary Figure 5.

Fig. 4 Developing a novel signature, $\operatorname{Sig}_{\text {evo }}$, for drug repositioning. a Schematic of the stepwise process of liver cancer initiation and progression. b Preliminary screening of developmental stage-associated genes by Random Forests algorithm. $\mathbf{c}$ The expression pattern of the 'ascending' module discerned by WGCNA analysis (left) and the enriched biological processes determined by hypergeometric test (right). d The expression pattern of the 'descending' module (left) and the enriched biological processes (right). e The performance evaluation of the $\operatorname{Sig}_{\text {evo }}$ for discerning the difference between tumor and normal tissues based on RNA sequencing cohorts (left) 
and microarray cohorts (right). f The association between the Sigevo and the clinical phenotype of prognosis. Color toward gray indicates no statistical significance. $\mathbf{g}$ The association between the $\operatorname{Sig}_{\text {evo }}$ and fibrosis-related phenotype suggested by ROC curve. $\mathbf{h}$ The association between $\mathrm{Sig}_{\mathrm{evo}}$ and CCl4-induced expression changes in liver tissues of mice. The enrichment scores and statistical significance were determined by gene set enrichment analysis. $\mathbf{i}$ The association between Sig $_{\text {evo }}$ and DEN-induced expression changes in liver tissues of rats.

Fig. 5 Identifying HHT as repositioning candidate with in vitro tumor killing activity. a Results of best practice approach-based computational drug repositioning using $\operatorname{Sig}_{\text {evo }}$ as query signature. Top ranked 10 compounds with highest reversal potency were illustrated in the right panel. b Enrichment of HCC agents in compounds with reversal potency (XSum score < 0). The statistical significance was determined based on the null distribution formed by 10,000 permutations. c 2D (left) and 3D (right) chemical structure of homoharringtonine (HHT). Data was derived from DrugBank. d Comparison of distribution of compound activity between HHT and three different drug categories, including chemotherapy ( $\mathrm{n}=45$ compounds), targeted cancer agents ( $\mathrm{n}=419$ compounds), and non-oncology ( $\mathrm{n}=362$ compounds). The $\mathrm{IC}_{50}$ value of each drug category in each cell line $(n=482)$ was determined through calculating the median $\mathrm{IC}_{50}$ value across all the compounds in this category. e The drug sensitivity data of HHT (achieved from PRISM dataset) across different HCC cell lines. The drug sensitivities of two HCC agents in the first-line (sorafenib and lenvatinib) and one HCC agent in the second-line (regorafenib) were also illustrated for comparison. Areas with different colors denote the interquartile range of median $\mathrm{IC}_{50}$ values of compounds within different drug categories. f Long-term cell proliferation assay for testing the anti-tumor activity of HHT across 11 liver cancer cell lines. Of these, five cell lines have not been profiled by PRISM for the sensitivity to HHT. $\mathbf{g}$ Therapeutic potency of the co-administration of HHT and sorafenib across 11 cell lines. h Two representative surface plots showing the overall synergistic (left) and antagonistic 
1152 (right) effects of the combination treatments.

1153

1154 Fig. 6 Assessing the anti-fibrotic effects of HHT. a Differential expression of nine 1155 fibrosis-associated genes between HHT-treated and HHT-untreated LX-2 cells. b 1156 Quantitative real-time PCR-based mRNA expression level of collagen I (upper) and $1157 \alpha$-SMA (lower) of LX-2 cells treated with gradient concentrations of HHT for 6 hours. 1158 c Western blot-based protein expression level of collagen I and $\alpha$-SMA of LX-2 cells 1159 treated with gradient concentrations of HHT for 24 hours. d Representative images of 1160 immunofluorescence stainings of LX-2 cells with antibody against collagen I (upper) 1161 and $\alpha$-SMA (lower). 


\section{Computational drug discovery and repurposing}

Collect disease samples for obtaining molecular profiles (e.g., liver cancer)

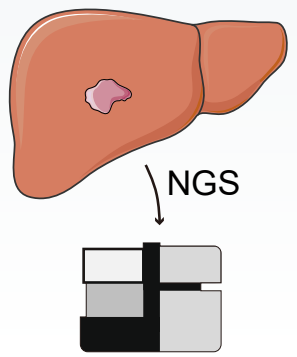

\section{Validation for computational results}

Approved for marketing

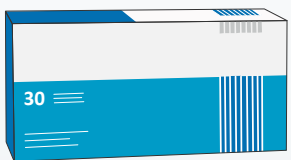

Clinical trials

(Phase I/II/III)

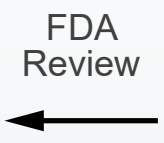

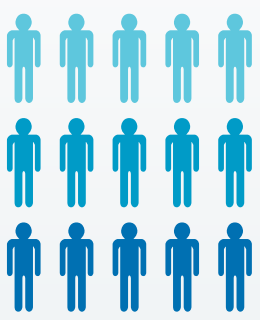

Establish disease gene expression signature

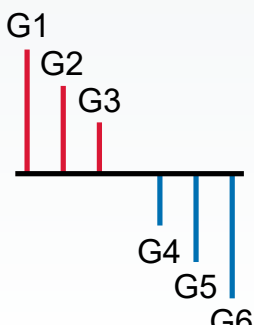

G6
Identify compounds that antagonize the disease signature

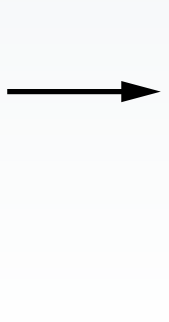

HCC gene signature

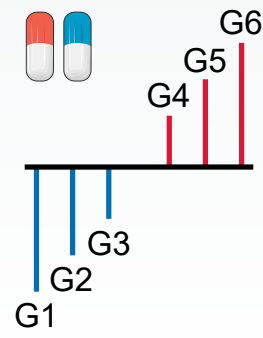

Compound signature

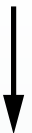

Preclinical evaluation

In vitro

In vivo

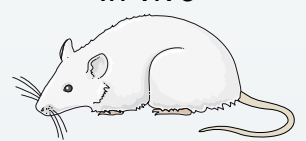


a

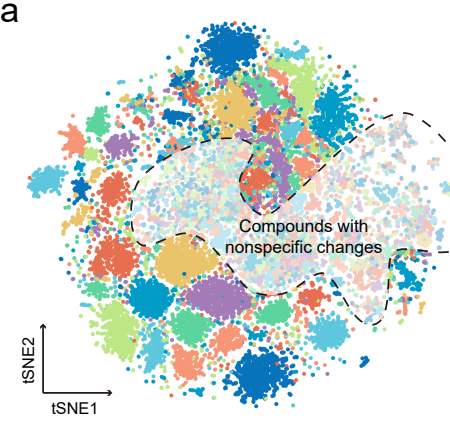

e

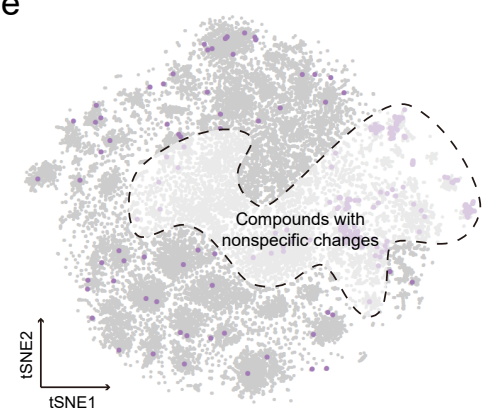

f

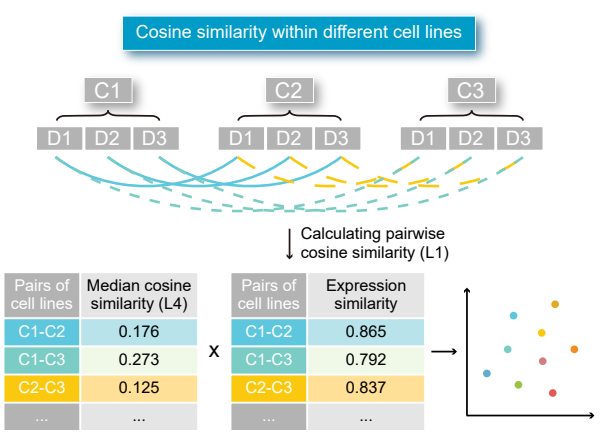

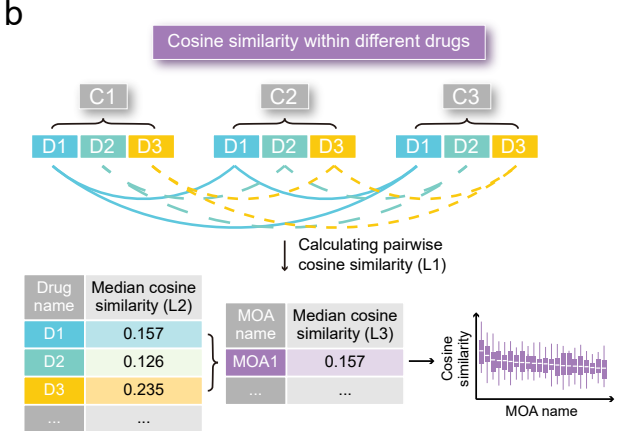

C

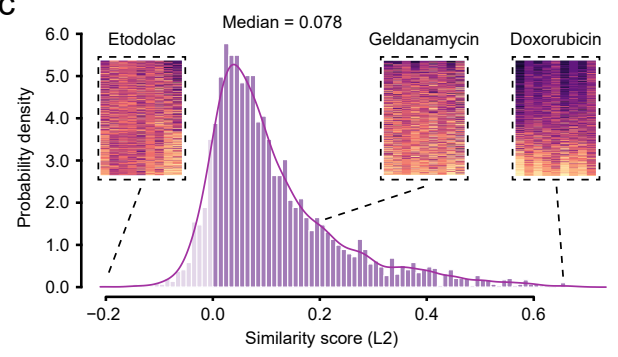

g

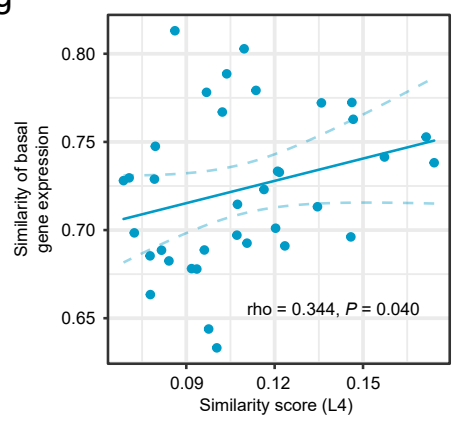

$d$

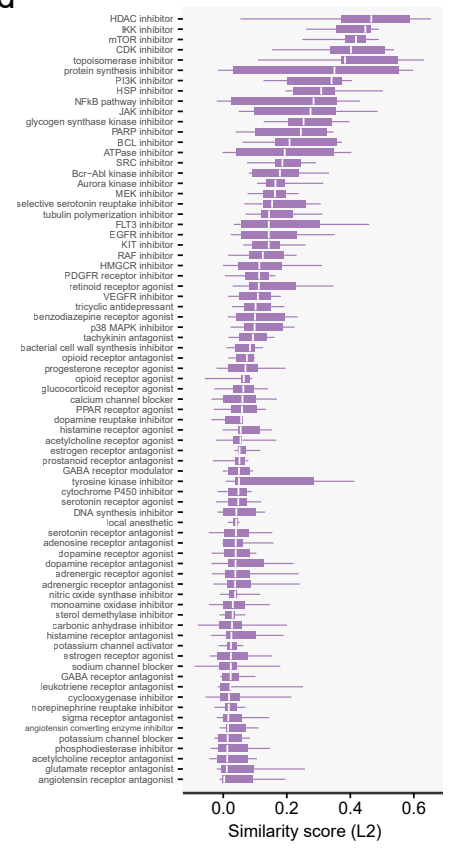

h
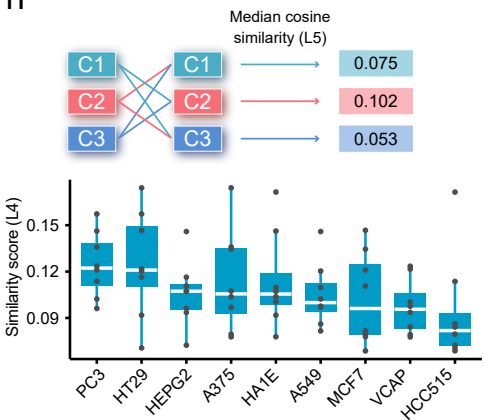


\section{$\downarrow$}

Four query signatures

- Sig1 (LIRI, RNAseq)

- Sig2 (GSE54236, Array)

- Sig3 (Gastro, Ref.11)

- Sig4 (NC, Ref.12)

Two benchmarking standards

- AUC-based standard

- KS-based standard

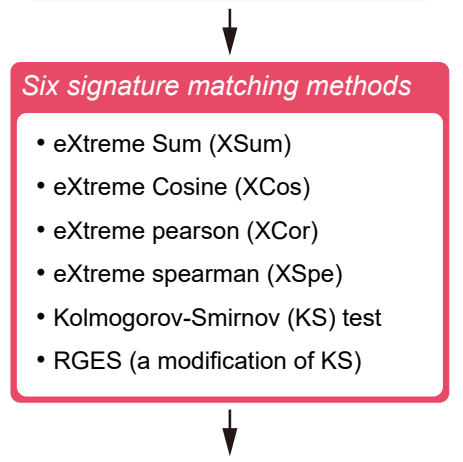

Identifying the optimal method and parameter for subsequent analyses
Two query signatures

- Sig1 (LIRI, RNAseq)

- Sig2 (GSE54236, Array)

One optimal method

r.X
Two benchmarking standards

- AUC-based standard

- KS-based standard

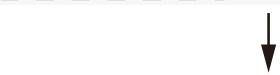

Two approaches for obtaining query signatures with different gene numbers

Iterative fold change- I | based approach
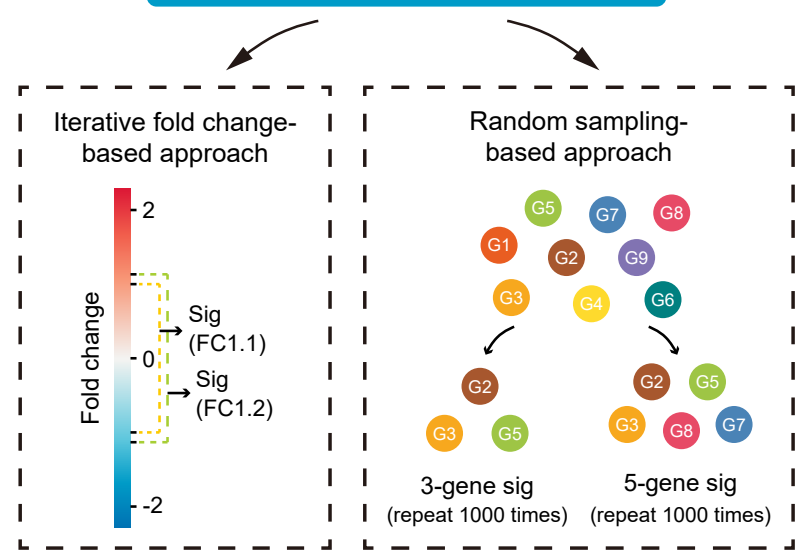

b
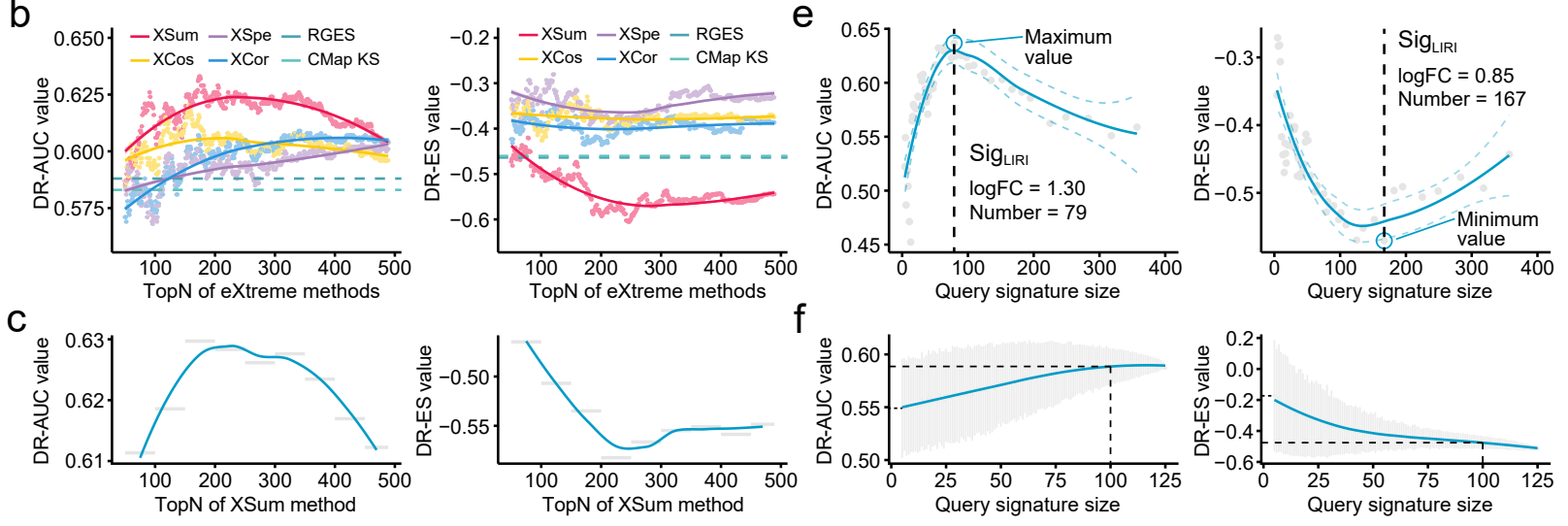
a

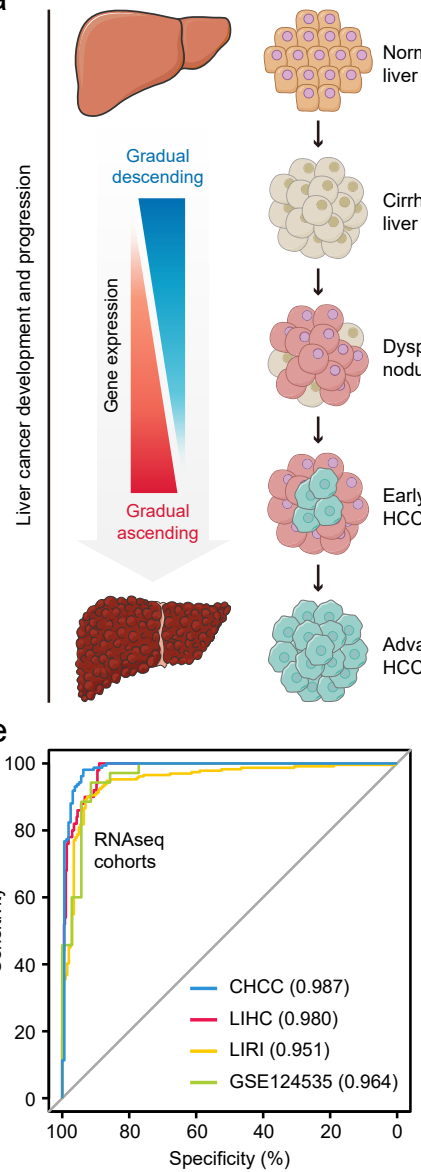

g

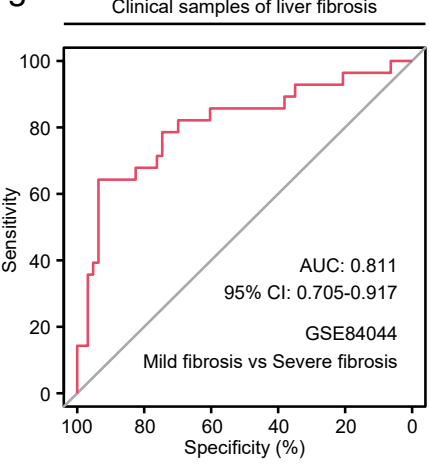

b
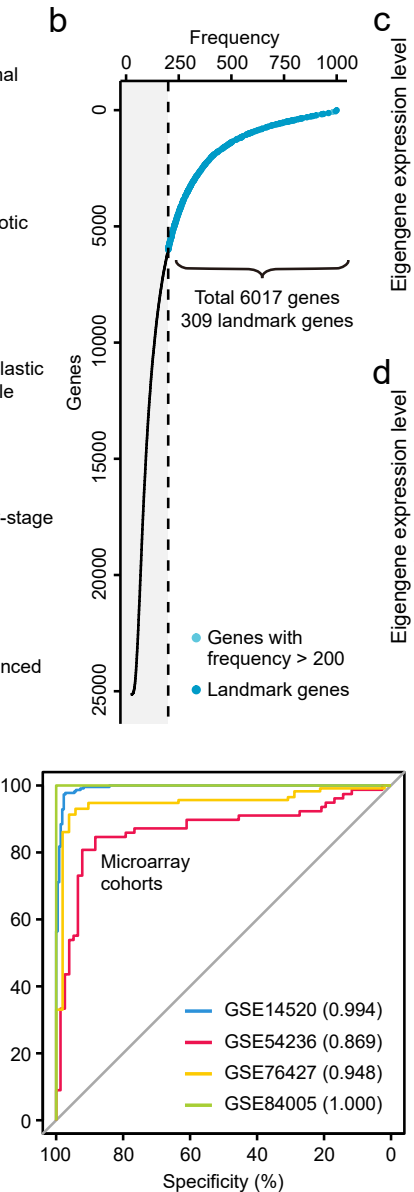

h

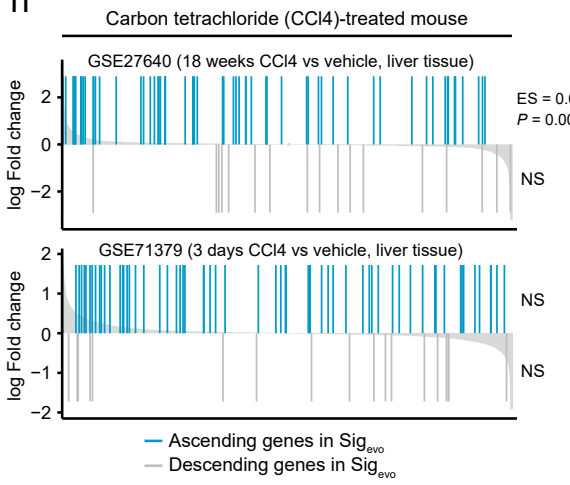

$f$
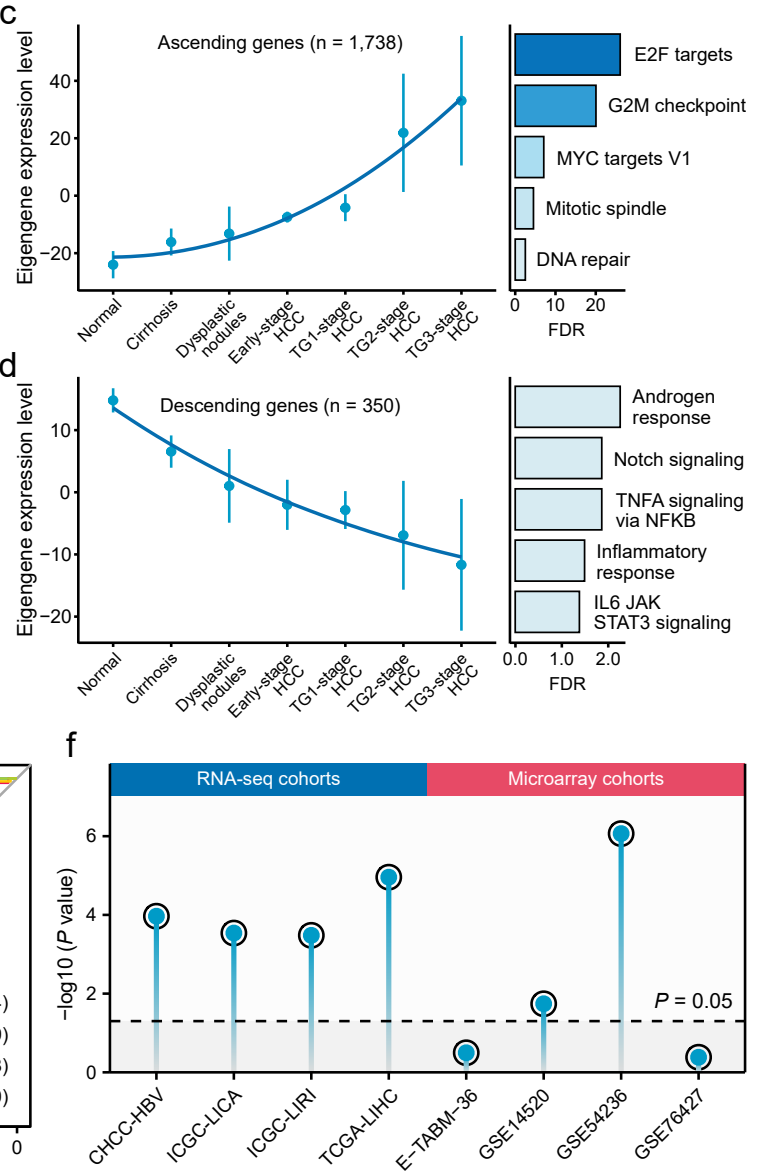

i
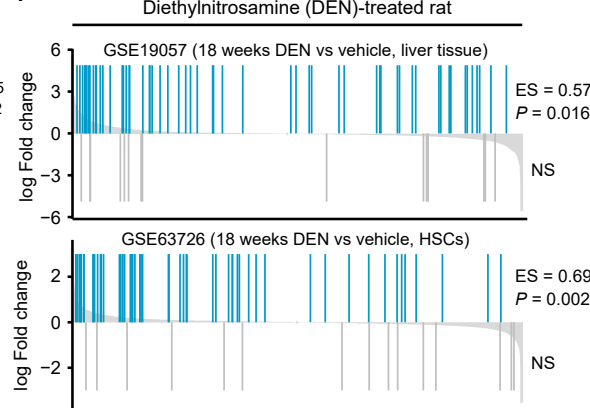

- Ascending genes in $\mathrm{Sig}_{\text {evo }}$

- Descending genes in $\mathrm{Sig}_{\text {evo }}$ 


\section{a}

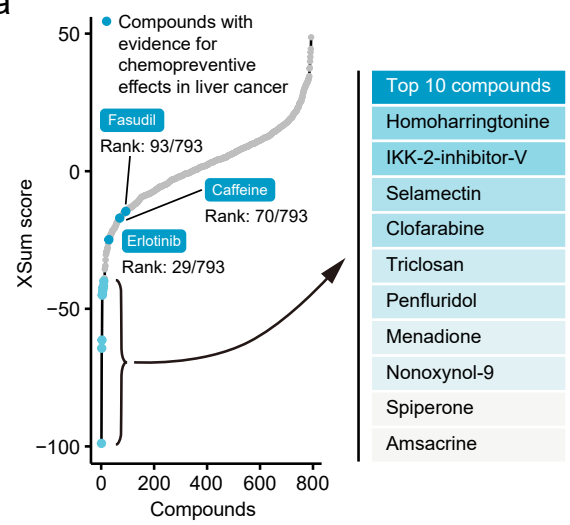

b

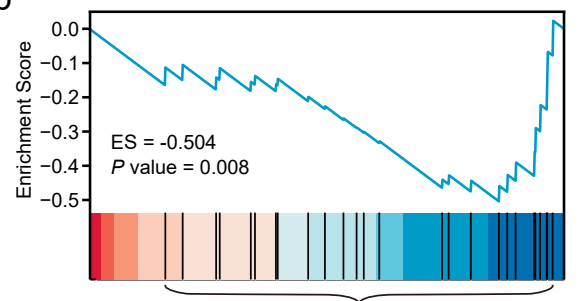

HCC agents
C
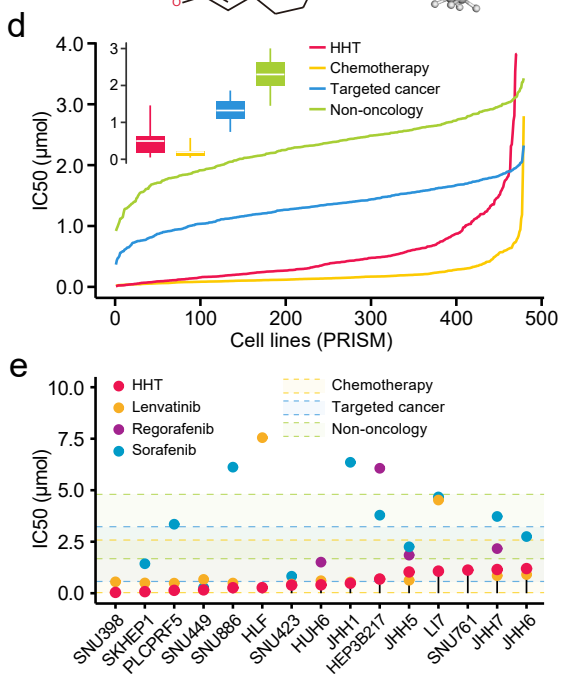

$\mathrm{h}$

449 (HSA)

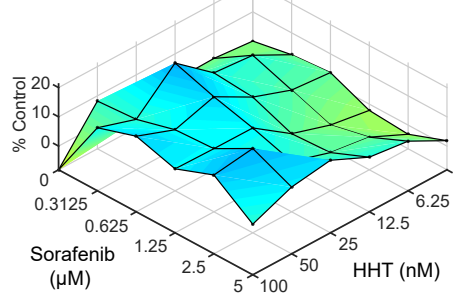

$\mathrm{HHT}(\mathrm{nM})$

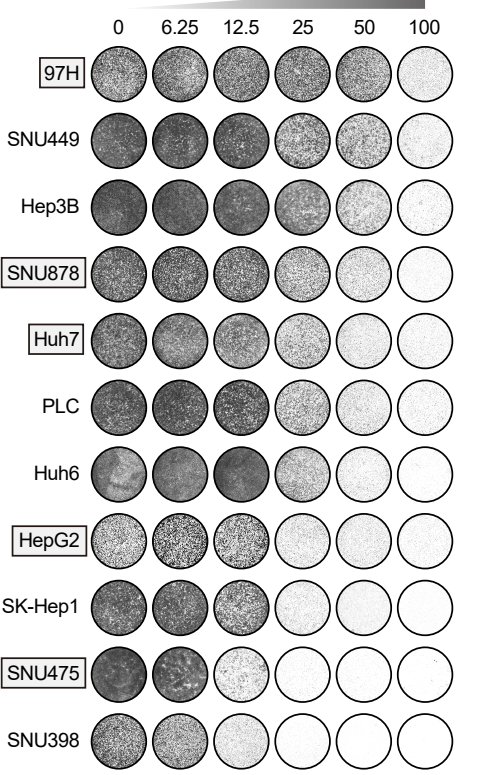

$\square$ Cell lines without previous

$\square$ HHT response data in PRISM

HepG2 (Bliss)

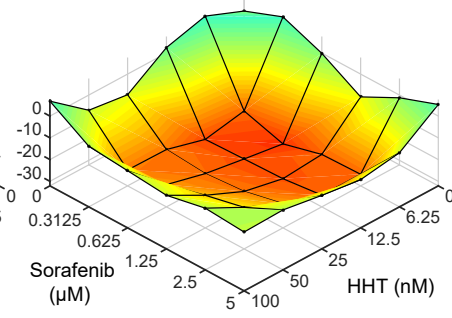

g

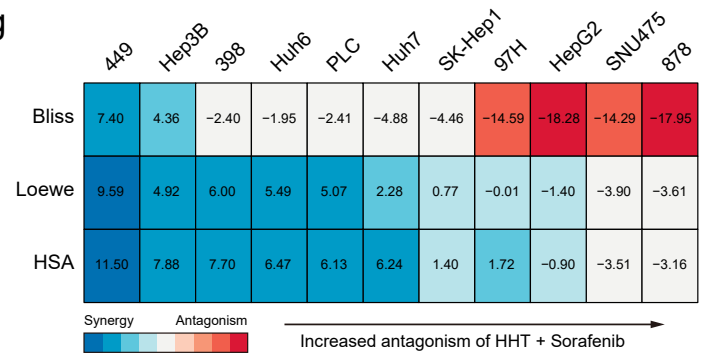


a

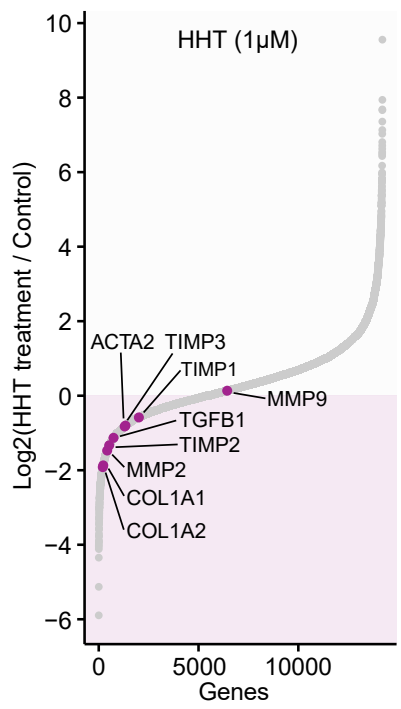

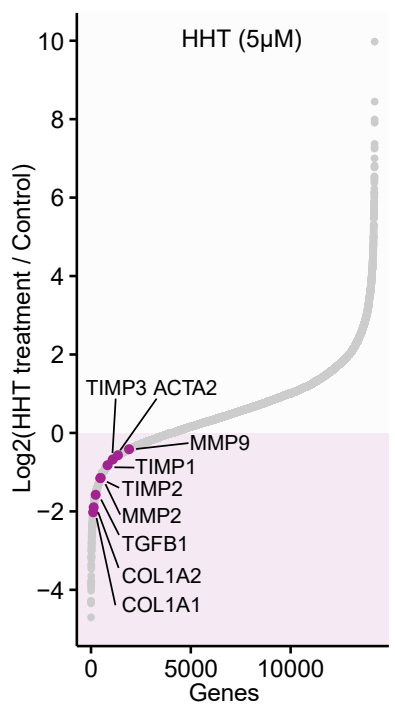

C

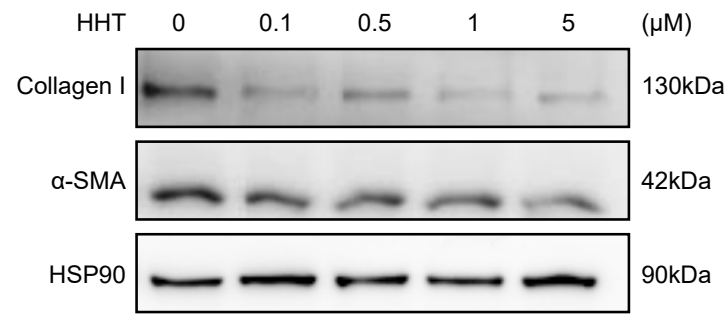

d
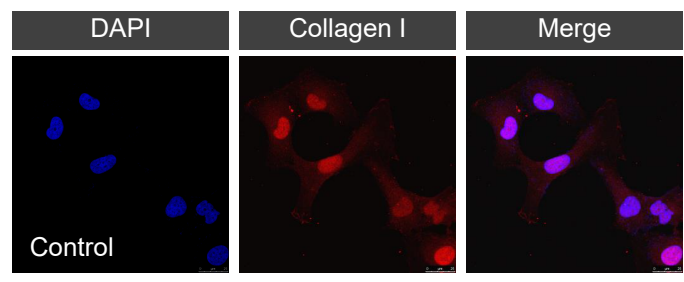

b

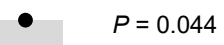

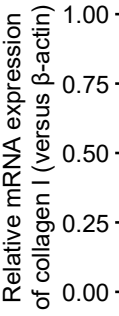

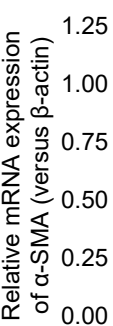

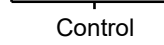

$\mathrm{HHT}$
$(0.1 \mu \mathrm{M})$

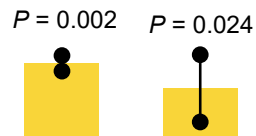

$P=0.002$
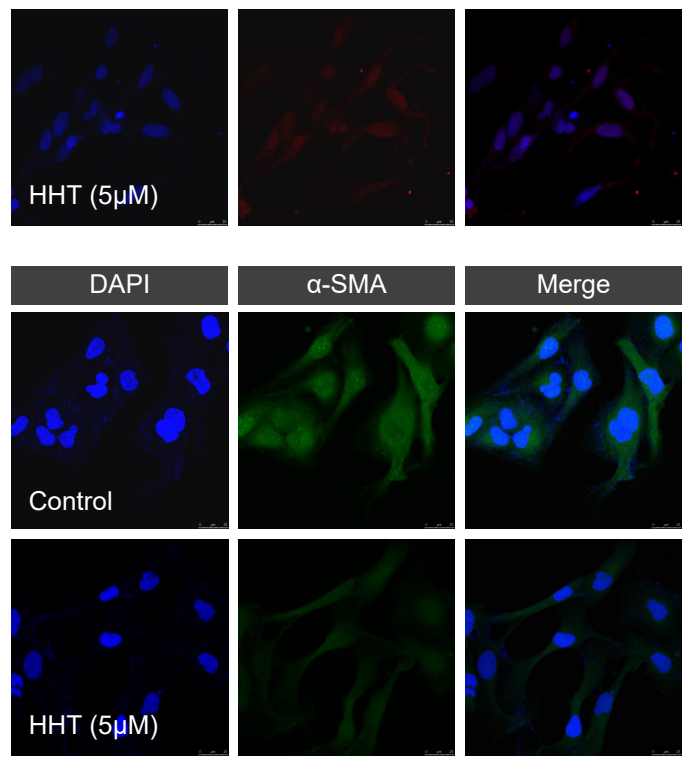

Control

HHT

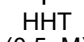

HHT

$\mathrm{HHT}$
$(5 \mu \mathrm{M})$ 